\title{
Neutrino mixing by modifying the Yukawa coupling structure of constrained sequential dominance
}

\author{
Joy Ganguly $^{*}$ and Raghavendra Srikanth Hundi ${ }^{\dagger}$ \\ Department of Physics, Indian Institute of Technology Hyderabad, Kandi-502 285, India
}

(Received 1 July 2020; accepted 12 January 2021; published 8 February 2021)

\begin{abstract}
In the constrained sequential dominance (CSD), tri-bimaximal mixing (TBM) pattern in the neutrino sector has been explained, by proposing a certain Yukawa coupling structure for the right-handed neutrinos of the model. However, from the current experimental data it is known that the values of neutrino mixing angles are deviated from the TBM values. In order to explain this neutrino mixing, we first propose a phenomenological model where we consider Yukawa couplings which are modified from that of CSD. Essentially, we add small complex parameters to the Yukawa couplings of CSD. Using these modified Yukawa couplings, we demonstrate that neutrino mixing angles can deviate from their TBM values. We also construct a model, based on a flavor symmetry, in order to justify the modified form of Yukawa couplings of our work.
\end{abstract}

DOI: 10.1103/PhysRevD.103.035007

\section{INTRODUCTION}

From various experimental observations it is known that neutrinos have very small mass [1]. In a type I seesaw mechanism, through the mediation of heavy right-handed neutrinos, smallness of neutrino masses can be understood $[2,3]$. To test this mechanism at the LHC the mass of the right-handed neutrinos should be around $1 \mathrm{TeV}$. However, with $1 \mathrm{TeV}$ masses for right-handed neutrinos some tuning in the Yukawa couplings may be required in order to fit the tiny masses of neutrinos. Moreover, due to large number of seesaw parameters this mechanism may not be predicted from the experimental data. To alleviate the above mentioned problems, models based on sequential dominance $[4,5]$ with two right-handed neutrinos and one texture zero in the neutrino Yukawa matrix have been proposed [6,7]. These models are named as $\operatorname{CSD}(n)$, which we describe briefly below.

It is known that the neutrinos mix among them [1] and the current oscillation data [8] suggest that the neutrino mixing angles are close to the TBM pattern [9]. To explain these mixing angles in the models of $\operatorname{CSD}(n)$, the two right-handed neutrinos are proposed to have certain particular Yukawa couplings with the three lepton doublets. To be specific, the two right-handed neutrinos, up to proportionality factors, are

\footnotetext{
*ph18resch11009@iith.ac.in

rshundi@phy.iith.ac.in
}

Published by the American Physical Society under the terms of the Creative Commons Attribution 4.0 International license. Further distribution of this work must maintain attribution to the author(s) and the published article's title, journal citation, and DOI. Funded by SCOAP ${ }^{3}$. proposed to have the following Yukawa couplings: $(0,1,1)$ and $(1, n, n-2)$. Here, $n$ is a positive integer but can be taken to be real as well. For the case of $n=1$, the model predicts that the three mixing angles will take the following TBM values: $\sin \theta_{12}=\frac{1}{\sqrt{3}}, \sin \theta_{23}=\frac{1}{\sqrt{2}}, \sin \theta_{13}=0$. This case of $n=1$ is originally named as constrained sequential dominance (CSD), which was viable a decade ago. But this case has been ruled out when Daya Bay and RENO measured the $\theta_{13}$ and found it to be nonzero [10]. Among the other integer values for $n$, only the models with $n=3,4$ are compatible with the current neutrino oscillation data [7].

In this work, we study on a possibility where we consider modifications to model parameters of CSD and demonstrate that the neutrino observables from the oscillation data can be explained. As explained above that CSD is nothing but $\operatorname{CSD}(n=1)$ and hence the Yukawa couplings in this model are proportional to $(0,1,1)$ and $(1,1,-1)$. In the next section we will describe that with this particular form for Yukawa couplings, the mixing angles for neutrinos can be predicted to have the TBM values. Now, in order to get deviations in neutrino mixing angles away from the TBM values, we consider the Yukawa couplings of the two righthanded neutrinos to be proportional to $\left(\epsilon_{1}, 1+\epsilon_{2}, 1+\epsilon_{3}\right)$ and $\left(1+\epsilon_{4}, 1+\epsilon_{5},-1+\epsilon_{6}\right)$. Here, $\epsilon_{i}, i=1, \ldots, 6$, are complex numbers. By proposing above mentioned Yukawa couplings for neutrinos, we are considering here a phenomenological model. Now, in this phenomenological model, in the limit where all $\epsilon_{i} \rightarrow 0$, our model should give the results of CSD. As a result of this, we can expect that for small parametric values of $\epsilon_{i}$ we should get deviations in neutrino mixing angles away from the TBM values. The reason for considering all $\epsilon_{i}$ to be small is due to the fact that the observed mixing angles are close 
to the TBM values. After assuming $\epsilon_{i}$ to be small, we study if we can consistently fit the neutrino masses and mixing angles, whose values are obtained from oscillation data.

Like in the model of CSD, in our model also only two right-handed neutrinos are proposed. As a result of this, in our model, one neutrino would be massless and the other two can have nonzero masses. Hence, in this model, we will show that only normal hierarchy is possible for neutrino masses. We can fit the nonzero masses of our model to square root of solar $\left(\sqrt{\Delta m_{\mathrm{sol}}^{2}}\right)$ and atmospheric $\left(\sqrt{\Delta m_{\mathrm{atm}}^{2}}\right)$ mass squared differences. From the global fits to neutrino oscillation data we can see that there is a hierarchy between $\Delta m_{\text {sol }}^{2}$ and $\Delta m_{\mathrm{atm}}^{2}$ [8]. In fact, from the results of Ref. [8], one can notice that $\frac{\Delta m_{\text {sol }}^{2}}{\Delta m_{\text {atm }}^{2}} \sim \sin ^{2} \theta_{13} \approx 2 \times 10^{-2}$. Because of this, we take $\sqrt{\frac{\Delta m_{\text {sol }}^{2}}{\Delta m_{\text {atm }}^{2}}}$ and $\sin \theta_{13}$ to be small, whose values can be around 0.15 .

As mentioned above, in our work, we are modifying the neutrino Yukawa couplings of CSD model by introducing small complex $\epsilon_{i}$ parameters. To be consistent with the oscillation data, we assume that the magnitude of real and imaginary parts of $\epsilon_{i}$ to be less than or of the order of $\sqrt{\frac{\Delta m_{\text {sol }}^{2}}{\Delta m_{\mathrm{atm}}^{2}}} \sim \sin \theta_{13}$. After assuming this, we diagonalize the seesaw formula for active neutrinos in our model, by following an approximation procedure, where we expand the seesaw formula in power series of $\epsilon_{i}$. Recently, this kind of diagonalization procedure has been used in a different neutrino mass model [11]. In the context of this present work, the usage and relevance of this diagonalization procedure have been described in Secs. III and IV. Following this diagonalization procedure, we derive expressions for neutrino masses and mixing angles in terms of $\epsilon_{i}$. We show that by keeping terms up to first order in $\epsilon_{i}$ of our analysis, we get $\sin \theta_{13}$ and $\sin \theta_{23}-\frac{1}{\sqrt{2}}$ to be nonzero but $\sin \theta_{12}-\frac{1}{\sqrt{3}}$ is found to be undetermined. In order to know if $\sin \theta_{12}-\frac{1}{\sqrt{3}}$ can be determined, we compute expressions in our analysis up to second order in $\epsilon_{i}$. Thereafter we demonstrate that $\sin \theta_{12}-\frac{1}{\sqrt{3}}$ can also be determined by $\epsilon_{i}$ parameters. Using the analytic expressions for neutrino masses and mixing angles, in order to be compatible with current neutrino oscillation data, we present numerical results and also demonstrate that the assumptions made in our diagonalization procedure are viable.

We study the above described work in a phenomenological model, where the neutrino Yukawa couplings of this model are modified from that of CSD model. One would like to know how such modified form for Yukawa couplings could be possible in our model. In order to address this point, toward the end of this paper, we construct a model, based on symmetry groups, where we explain the smallness of $\epsilon_{i}$ parameters and also justify the structure of Yukawa couplings of our phenomenological model. In order for this model to explain the structure of Yukawa couplings, the scalar fields proposed in this model need to acquire vacuum expectation values (vevs) with hierarchically different magnitudes. To explain the hierarchy in the vev of these scalar fields, we analyze the scalar potential among these fields and give one solution to this problem.

Deviations from TBM pattern has been studied in sequential dominance models [5], where neutrino masses and mixing angles are computed in a general framework of type I seesaw model and then these results are applied to models which satisfy sequential dominance conditions. Here, our approach to the problem is different from that of Ref. [5]. In this work, we first modify the Yukawa coupling structure of CSD and then study the deviations from TBM pattern. Moreover, our analysis is also different from that of Ref. [5].

The paper is organized as follows. In the next section we describe sequential dominance and the CSD model. In Sec. III, we describe our phenomenological model and also explain the approximation procedure for diagonalizing the seesaw formula for neutrinos of this model. Using this approximation procedure we demonstrate that the neutrino mixing angles in our model deviate away from the TBM pattern. In the same section, we compute expressions for neutrino masses and mixing angles up to first order in our approximation scheme. Second order corrections to the above mentioned neutrino observables have been computed in Sec. IV. In Sec. V, we give numerical results where we demonstrate that our analytic expressions can fit the current neutrino oscillation data. In Sec. VI, we construct a model in order to justify the structure of Yukawa couplings of our phenomenological model. We conclude in the last section. In Appendix A, we have given detailed expressions related to the second order corrections to the neutrino observables. In Appendix B, we analyze the scalar potential of our model in order to explain the hierarchy in the vevs of the scalar fields.

\section{SEQUENTIAL DOMINANCE AND CSD}

The idea for CSD is motivated from sequential dominance, which is briefly described below. Consider a minimal extension to the standard model, where the additional fields are three singlet right-handed neutrinos. After electroweak symmetry breaking, charged leptons and neutrinos acquire mixing mass matrices. We can consider a basis in which both charged leptons and right-handed neutrinos have been diagonalized. In this basis, the mass matrix for right-handed neutrinos and the mixing mass matrix between left- and right-handed neutrinos can be written, respectively, as

$$
M_{R}=\left(\begin{array}{ccc}
M_{\mathrm{atm}} & 0 & 0 \\
0 & M_{\mathrm{sol}} & 0 \\
0 & 0 & M_{\mathrm{dec}}
\end{array}\right), \quad m_{D}=\left(\begin{array}{ccc}
d & a & a^{\prime} \\
e & b & b^{\prime} \\
f & c & c^{\prime}
\end{array}\right)
$$


In the equation for $m_{D}$, elements such as $a, b, c$, etc can be viewed as neutrino Yukawa coupling multiplied by vev of the Higgs field. Assuming that the masses for right-handed neutrinos are much larger than the elements of Dirac mass matrix, the seesaw formula for active neutrinos would be

$$
m_{\nu}=m_{D} M_{R}^{-1} m_{D}^{T}
$$

From the seesaw formula we get three masses for active neutrinos, which may be denoted by $m_{1}, m_{2}$, and $m_{3}$. The objective of sequential dominance is to achieve $m_{1} \ll m_{2} \ll m_{3}$, and thereby the model can predict normal mass hierarchy for neutrinos. In order to achieve this objective of sequential dominance, following assumptions on the masses of right-handed neutrinos and the elements of the Dirac mass matrix have been made [4,5]

$M_{\mathrm{atm}} \ll M_{\mathrm{sol}} \ll M_{\mathrm{dec}}, \frac{\left|e^{2}\right|,\left|f^{2}\right|,|e f|}{M_{\mathrm{atm}}} \gg \frac{x y}{M_{\mathrm{sol}}} \gg \frac{x^{\prime} y^{\prime}}{M_{\mathrm{dec}}}$

Here, $x, y \in a, b, c$ and $x^{\prime}, y^{\prime} \in a^{\prime}, b^{\prime}, c^{\prime}$.

With the above mentioned assumptions of sequential dominance, leading order expressions for neutrino masses and mixing angles have been computed in Ref. [12]. Using these expressions, the following set of conditions on the model parameters have been proposed, in order to obtain the TBM pattern for neutrino mixing angles [6].

$$
\begin{aligned}
& |a|=|b|=|c|, \quad|d|=0, \quad|e|=|f|, \\
& \phi_{b}^{\prime}=0, \quad \phi_{c}^{\prime}=\pi
\end{aligned}
$$

Here, $\phi_{b}^{\prime}$ and $\phi_{c}^{\prime}$ denote sum of a combination of phases of the elements in the Dirac mass matrix [6]. From the above mentioned conditions we can notice that the elements in the third column of $m_{D}$ and $M_{R}$ play no part in determining the TBM pattern for neutrino mixing angles. In fact, from the leading order expressions for neutrino masses and mixing angles given in Ref. [12], we can see that the third column elements of $m_{D}$ and $M_{R}$ determine only the lightest neutrino mass $m_{1}$. One can notice that $m_{1}$ is proportional to $\frac{1}{M_{\mathrm{dec}}}$. Now, in the limit where the value of $M_{\mathrm{dec}}$ tends to very large, we get $m_{1} \rightarrow 0$. In this limiting process, the third right-handed neutrino, whose mass is $M_{\mathrm{dec}}$, decouples from our theory. Since the current experimental data can be satisfied with $m_{1}=0$, in order to reduce the number of degrees of freedom in this model, we can decouple away the third column elements of $m_{D}$ and $M_{R}$. Essentially, in this process of decoupling, the number of right-handed neutrinos reduce from three to two in the above described model.

After performing the above mentioned decoupling, in the resultant model, to satisfy the conditions of Eq. (4), the Dirac and right-handed neutrino mass matrices can be taken, respectively, as [6]

$$
m_{D}=\left(\begin{array}{cc}
0 & a \\
e & a \\
e & -a
\end{array}\right), \quad M_{R}=\left(\begin{array}{cc}
M_{\mathrm{atm}} & 0 \\
0 & M_{\mathrm{sol}}
\end{array}\right)
$$

By plugging the above mentioned $m_{D}$ and $M_{R}$ in the seesaw formula of Eq. (2), we can check that the $m_{\nu}$ can be diagonalized as

$$
\begin{aligned}
U_{\mathrm{TBM}}^{T} m_{\nu} U_{\mathrm{TBM}} & =\left(\begin{array}{ccc}
0 & 0 & 0 \\
0 & \frac{3 a^{2}}{M_{\mathrm{sol}}} & 0 \\
0 & 0 & \frac{2 e^{2}}{M_{\mathrm{atm}}}
\end{array}\right), \\
U_{\mathrm{TBM}} & =\left(\begin{array}{ccc}
\sqrt{\frac{2}{3}} & \frac{1}{\sqrt{3}} & 0 \\
-\frac{1}{\sqrt{6}} & \frac{1}{\sqrt{3}} & \frac{1}{\sqrt{2}} \\
\frac{1}{\sqrt{6}} & -\frac{1}{\sqrt{3}} & \frac{1}{\sqrt{2}}
\end{array}\right)
\end{aligned}
$$

From the unitary matrix $U_{\mathrm{TBM}}$, one can extract the three neutrino mixing angles and we see that they will have the TBM values.

We have demonstrated above that in a model with two right-handed neutrinos, which is motivated by sequential dominance, TBM pattern for neutrino mixing is possible. This has been named as CSD [6]. One can notice that in this process of obtaining TBM pattern, the columns of Dirac mass matrix need to be aligned in some particular directions. This problem of alignment has been addressed in a supersymmetric model which has some flavor symmetries and flavon fields [6].

\section{OUR MODEL AND DEVIATIONS FROM TBM PATTERN}

In the previous section we have described on how CSD can predict TBM pattern for neutrino mixing angles. Since this pattern is currently ruled out, we need to modify the model of CSD. To achieve this, we initially consider a phenomenological model where the field content is same as that of CSD. But the difference between our model and the CSD is that we propose a modified structure for Dirac mass matrix, which is given below.

$$
\begin{aligned}
m_{D}^{\prime} & =m_{D}+\Delta m_{D}, \quad m_{D}=\left(\begin{array}{cc}
0 & a \\
e & a \\
e & -a
\end{array}\right), \\
\Delta m_{D} & =\left(\begin{array}{ll}
e \epsilon_{1} & a \epsilon_{4} \\
e \epsilon_{2} & a \epsilon_{5} \\
e \epsilon_{3} & a \epsilon_{6}
\end{array}\right) .
\end{aligned}
$$

Here, $\epsilon_{i}, i=1, \ldots, 6$, are complex parameters. At this stage we are suggesting the above form for Dirac mass matrix, 
purely from phenomenological point of view. We justify this form of matrix by constructing a model for this in Sec. VI. Regarding the Dirac mass matrix, we have explained in the previous section that the elements of this matrix should be viewed as a product of neutrino Yukawa couplings and vev of the Higgs field. As a result of this, the above Dirac mass matrix corresponds to the fact that the Yukawa couplings of the two right-handed neutrinos are proportional to $\left(\epsilon_{1}, 1+\epsilon_{2}, 1+\epsilon_{3}\right)$ and $\left(1+\epsilon_{4}, 1+\epsilon_{5},-1+\epsilon_{6}\right)$. As we have argued in Sec. I, with this form for Yukawa couplings we should expect to get deviations for neutrino mixing angles away from the TBM values.

$$
U_{\mathrm{PMNS}}=\left(\begin{array}{c}
c_{12} c_{13} \\
-s_{12} c_{23}-c_{12} s_{23} s_{13} e^{i \delta_{C P}} \\
s_{12} s_{23}-c_{12} c_{23} s_{13} e^{i \delta_{C P}}
\end{array}\right.
$$

Here, $c_{i j}=\cos \theta_{i j}$ and $s_{i j}=\sin \theta_{i j}$. As explained above that in our model, with the form for $m_{D}^{\prime}$ of Eq. (7), we should get deviations in the neutrino mixing angles away from the TBM values. As a result of this, we should expect $s_{13}, s_{12}-1 / \sqrt{3}$ and $s_{23}-1 / \sqrt{2}$ to become nonzero. In order to simplify our calculations, we parametrize $s_{12}$ and $s_{23}$ as

$$
s_{12}=\frac{1}{\sqrt{3}}(1+r), \quad s_{23}=\frac{1}{\sqrt{2}}(1+s)
$$

The parametrization we have considered for neutrino mixing angles is similar to that proposed in Refs. [14]. For a different parametrization of these neutrino mixing angles, see Ref. [15]. We have known the $3 \sigma$ ranges for the square of the sine of the neutrino mixing angles, which are obtained from the global fits to oscillation data [8]. From these $3 \sigma$ ranges, we can find the corresponding ranges for $r$ and $s$, which are found, respectively, as: $\left(-8.8 \times 10^{-2}\right.$, $\left.2.5 \times 10^{-2}\right)$ and $\left(-8.2 \times 10^{-2}, 0.13\right)$. The corresponding allowed range for $s_{13}$ is found to be narrow, whose values are around 0.15 . From the above mentioned ranges, we can notice that the values for $r$ and $s$ are less than or of the order of $s_{13}$. As explained before that $r, s$ and $s_{13}$ will become nonzero in our model, if we allow nonzero values for $\epsilon_{i}$ parameters in $m_{D}^{\prime}$. As a result of this, to be consistent with our analysis, we assume that the real and imaginary parts of $\epsilon_{i}$ to be less than or of the order of $s_{13}$.

As described previously, seesaw formula for active neutrinos in our model is given by Eq. (8) and this matrix should be diagonalized by $U_{\text {PMNS }}$. The relation for this diagonalization can be written as

$$
m_{\nu}^{d} \equiv U_{\mathrm{PMNS}}^{T} m_{\nu}^{s} U_{\mathrm{PMNS}}=\operatorname{diag}\left(m_{1}, m_{2}, m_{3}\right)
$$

As explained above that in our model, the form for Dirac mass matrix is given by $m_{D}^{\prime}$ and hence the seesaw formula for active neutrinos is

$$
m_{\nu}^{s}=m_{D}^{\prime} M_{R}^{-1}\left(m_{D}^{\prime}\right)^{T} .
$$

Since we are in a basis where charged leptons are diagonalized, this seesaw formula should be diagonalized by Pontecorvo-Maki-Nakagawa-Sakata (PMNS) matrix. The PMNS matrix can be parametrized by the neutrino mixing angles and the $C P$ violating Dirac phase $\delta_{C P}$. We follow the PDG convention for this parametrization [13], which is given below.

$$
\left.\begin{array}{cc}
s_{12} c_{13} & s_{13} e^{-i \delta_{C P}} \\
c_{12} c_{23}-s_{12} s_{23} s_{13} e^{i \delta_{C P}} & s_{23} c_{13} \\
-c_{12} s_{23}-s_{12} c_{23} s_{13} e^{i \delta_{C P}} & c_{23} c_{13}
\end{array}\right)
$$

Here, the matrices $m_{\nu}^{s}$ and $U_{\mathrm{PMNS}}$ depend on variables $\epsilon_{i}, r$, $s$ and $s_{13}$, which are small. As a result of this, we can expand $m_{\nu}^{s}$ and $U_{\text {PMNS }}$ as power series in terms of these small variables. First we expand $m_{\nu}^{s}$ and $U_{\text {PMNS }}$ up to first order in $\epsilon_{i}, r, s$ and $s_{13}$. After doing that one can see that $m_{\nu}^{d}$ need not be in diagonal form. But, since we expect this to be of diagonal form, we demand that the off-diagonal elements of $m_{\nu}^{d}$ to be zero. Thereby we get three relations among $\epsilon_{i}, r, s$, and $s_{13}$. Solving these relations, we can determine $\epsilon_{i}$ in terms of $r, s$, and $s_{13}$. Now, from the diagonal elements of $m_{\nu}^{d}$ we get expressions for the three neutrino masses in terms of model parameters. We follow the above described methodology for diagonalizing the seesaw formula of our model. However, while doing so, one needs to take care of the small numbers that may arise due to hierarchy in neutrino masses. Discussion related to this is explained below.

In the limit where $\epsilon_{i}, r, s$, and $s_{13}$ tend to zero, from Eq. (11) we get the leading order expressions for neutrino masses, which are given below.

$$
m_{1}=0, \quad m_{2}=\frac{3 a^{2}}{M_{\mathrm{sol}}}, \quad m_{3}=\frac{2 e^{2}}{M_{\mathrm{atm}}} .
$$

The above result agree with that of CSD which is given in Sec. II. Here, up to the leading order, the lightest neutrino mass $m_{1}$ is zero. However, we will show later that even at subleading orders, $m_{1}$ is still zero. This result is due to the consequence of the fact that in our model we have proposed only two right-handed neutrinos. As a result of this, neutrino masses in our model can only have normal mass hierarchy. Due to this, we can fit the expressions for $m_{2}$ and $m_{3}$ to square root of solar $\left(\sqrt{\Delta m_{\text {sol }}^{2}}\right)$ and atmospheric $\left(\sqrt{\Delta m_{\mathrm{atm}}^{2}}\right)$ mass squared differences, respectively. 
Although the expressions in Eq. (12) are valid at leading order, at subleading orders, expressions for $m_{2}$ and $m_{3}$ get corrections which are proportional to $\epsilon_{i}, r, s$, and $s_{13}$. Since $\epsilon_{i}, r, s$, and $s_{13}$ are small values, when we fit the expressions for $m_{2}$ and $m_{3}$ to $\sqrt{\Delta m_{\text {sol }}^{2}}$ and $\sqrt{\Delta m_{\mathrm{atm}}^{2}}$ respectively, we except to have the following order of estimations.

$$
\frac{a^{2}}{M_{\mathrm{sol}}} \sim \sqrt{\Delta m_{\mathrm{sol}}^{2}}, \quad \frac{e^{2}}{M_{\mathrm{atm}}} \sim \sqrt{\Delta m_{\mathrm{atm}}^{2}} .
$$

We use the above mentioned order of estimations in the diagonalization process of the seesaw formula of our model. Regarding this, a point to be noticed here is that, from the global fits to neutrino oscillation data [8], a hierarchy is found between $\Delta m_{\mathrm{sol}}^{2}$ and $\Delta m_{\mathrm{atm}}^{2}$. In fact, from the results of Ref. [8], one can notice that $\sqrt{\frac{\Delta m_{\text {sol }}^{2}}{\Delta m_{\text {atm }}^{2}}} \sim s_{13}$. This would imply that, in our model, $m_{2} / m_{3} \sim s_{13}$. One needs to incorporate the above mentioned order of estimation in the diagonalization process of the seesaw formula of our model. In order to incorporate this, we reexpress Eq. (11) as

$$
\begin{aligned}
\frac{1}{\sqrt{\Delta m_{\mathrm{atm}}^{2}}} m_{\nu}^{d} & \equiv \frac{1}{\sqrt{\Delta m_{\mathrm{atm}}^{2}}} U_{\mathrm{PMNS}}^{T} m_{\nu}^{s} U_{\mathrm{PMNS}} \\
& =\operatorname{diag}\left(\frac{m_{1}}{\sqrt{\Delta m_{\mathrm{atm}}^{2}}}, \frac{m_{2}}{\sqrt{\Delta m_{\mathrm{atm}}^{2}}}, \frac{m_{3}}{\sqrt{\Delta m_{\mathrm{atm}}^{2}}}\right)
\end{aligned}
$$

Now, with the assumptions of Eq. (13), one can see that $\frac{1}{\sqrt{\Delta m_{\text {atm }}^{2}}} m_{\nu}^{d}$ can be expanded in power series of $\epsilon_{i}, r, s, s_{13}$, and $\sqrt{\frac{\Delta m_{\text {sol }}^{2}}{\Delta m_{\text {atm }}^{2}}}$. We explain below about this series expansion and also the results obtained from such expansion.

Up to first order in $\epsilon_{i}, m_{\nu}^{s}$ can be expanded as

$$
\begin{gathered}
m_{\nu}^{s}=m_{\nu(0)}^{s}+m_{\nu(1)}^{s}, \\
m_{\nu(0)}^{s}=m_{D} M_{R}^{-1} m_{D}^{T}, \\
m_{\nu(1)}^{s}=m_{D} M_{R}^{-1}\left(\Delta m_{D}\right)^{T}+\Delta m_{D} M_{R}^{-1} m_{D}^{T} .
\end{gathered}
$$

Similarly, up to first order in $r, s$, and $s_{13}$, the expansion for $U_{\text {PMNS }}$ is

$$
\begin{gathered}
U_{\mathrm{PMNS}}=U_{\mathrm{TBM}}+\Delta U \\
\Delta U=\left(\begin{array}{ccc}
-\frac{r}{\sqrt{6}} & \frac{r}{\sqrt{3}} & e^{-i \delta_{\mathrm{CP}}} s_{13} \\
\frac{-r+s}{\sqrt{6}}-\frac{e^{i \delta} \mathrm{CP}_{13}}{\sqrt{3}} & -\frac{r+2 s+\sqrt{2} e^{i \delta \mathrm{CP} s_{13}}}{2 \sqrt{3}} & \frac{s}{\sqrt{2}} \\
\frac{r+s}{\sqrt{6}}-\frac{e^{i \delta \mathrm{CP} s_{13}}}{\sqrt{3}} & \frac{r-2 s-\sqrt{2} e^{i \delta \mathrm{CP} s_{13}}}{2 \sqrt{3}} & -\frac{s}{\sqrt{2}}
\end{array}\right)
\end{gathered}
$$

Here, the form of $U_{\text {TBM }}$ can be seen in Eq. (6). After substituting Eqs. (15) and (17) in Eq. (14) and with the assumptions of Eq. (13), we can compute $\frac{1}{\sqrt{\Delta m_{\text {atm }}^{2}}} m_{\nu}^{d}$ up to first order in $\epsilon_{i}, r, s, s_{13}$, and $\sqrt{\frac{\Delta m_{\text {sol }}^{2}}{\Delta m_{\text {atm }}^{2}}}$. Terms up to first order in $\frac{1}{\sqrt{\Delta m_{\text {atm }}^{2}}} m_{\nu}^{d}$ are given below.

$$
\begin{aligned}
\frac{1}{\sqrt{\Delta m_{\mathrm{atm}}^{2}} m_{\nu}^{d}}= & \frac{1}{\sqrt{\Delta m_{\mathrm{atm}}^{2}}}\left(m_{\nu(0)}^{d}+m_{\nu(1)}^{d}\right), \\
m_{\nu(0)}^{d} & =\left(\begin{array}{ccc}
0 & 0 & 0 \\
0 & \frac{3 a^{2}}{M_{\mathrm{sol}}} & 0 \\
0 & 0 & \frac{2 e^{2}}{M_{\mathrm{atm}}}
\end{array}\right), \\
m_{\nu(1)}^{d} & =\left(\begin{array}{ccc}
x_{11}^{\prime} & x_{12}^{\prime} & x_{13}^{\prime} \\
x_{12}^{\prime} & x_{22}^{\prime} & x_{23}^{\prime} \\
x_{13}^{\prime} & x_{23}^{\prime} & x_{33}^{\prime}
\end{array}\right), \\
x_{11}^{\prime} & =0, \quad x_{12}^{\prime}=0, \\
x_{13}^{\prime} & =\frac{e^{2}}{\sqrt{6} M_{\mathrm{atm}}}\left[\sqrt{2}\left(2 \epsilon_{1}-\epsilon_{2}+\epsilon_{3}+2 s\right)-4 e^{i \delta_{\mathrm{CP}}} s_{13}\right], \\
x_{22}^{\prime} & =0, \\
x_{23}^{\prime} & =\frac{e^{2}}{\sqrt{3} M_{\mathrm{atm}}}\left[\sqrt{2}\left(\epsilon_{1}+\epsilon_{2}-\epsilon_{3}-2 s\right)-2 e^{i \delta_{\mathrm{CP}}} s_{13}\right], \\
x_{33}^{\prime} & =\frac{2 e^{2}}{M_{\mathrm{atm}}} \cdot\left(\epsilon_{2}+\epsilon_{3}\right)
\end{aligned}
$$

Now, equating the diagonal elements on both sides of Eq. (14), we get the expressions for the three neutrino masses, which are given below

$$
m_{1}=0, \quad m_{2}=\frac{3 a^{2}}{M_{\mathrm{sol}}}, \quad m_{3}=\frac{2 e^{2}}{M_{\mathrm{atm}}}+\frac{2 e^{2}\left(\epsilon_{2}+\epsilon_{3}\right)}{M_{\mathrm{atm}}}
$$

From the above equations we can see that only $m_{3}$ get correction at the first order level. Now, from the offdiagonal elements of Eq. (14), we get the following expressions.

$$
\epsilon_{1}=\sqrt{2} e^{i \delta_{\mathrm{CP}}} s_{13}, \quad \epsilon_{2}-\epsilon_{3}=2 s
$$

From the above two equations we can see that, in our model, $\sin \theta_{13}$ will be nonzero if we take $\epsilon_{1} \neq 0$. Similarly, $\sin \theta_{23}$ will deviate from its TBM value if we take either $\epsilon_{2}$ or $\epsilon_{3}$ to be nonzero. However, the deviation of $\sin \theta_{12}$ from its TBM value, which is quantified in terms of $r$, is undetermined at the first order level corrections to the diagonalization of our seesaw formula. As a result of this, the parameters $\epsilon_{4}, \epsilon_{5}$ and $\epsilon_{6}$ are undetermined at this level. We will show in the next section that these parameters can be determined in terms of neutrino mixing angles by 
considering second order level corrections to the diagonalization of our seesaw formula.

Results obtained in Eq. (21) are consistent with that in Partially CSD (PCSD) [16]. In the model of PCSD, the structure of neutrino Yukawa couplings is similar to that in our model. The Yukawa couplings in PCSD can be obtained from that of our model by taking $\epsilon_{1} \neq 0$ and all other $\epsilon_{i}$ to be zero. With this Yukawa coupling structure, in the PCSD model, it is shown that $\sin \theta_{13} \neq 0$ after assuming TBM values for $\sin \theta_{12}$ and $\sin \theta_{23}$. These results are obtained in PCSD model up to a leading order in $m_{2} / m_{3}$. Results in previous paragraph are also obtained up to this order. Although we have argued that the value of $r$ is undetermined up to this order, with out loss of generality, in the beginning of the calculations, we can assume TBM value for $\sin \theta_{12}$ and choose zero values for $\epsilon_{4}, \epsilon_{5}$ and $\epsilon_{6}$. In that case, we would still get the results of Eq. (21). Now if we choose zero values for $\epsilon_{2}$ and $\epsilon_{3}$, that would imply TBM value for $\sin \theta_{23}$. Hence, results obtained in the previous paragraph are consistent with that of PCSD model. Moreover, it is to be noticed that the structure of our model and the results obtained in this work generalizes that of PCSD model. We have described above about the relevance of the relations in Eq. (21). It should be noted that, in our framework, these relations cannot be obtained without making the assumptions of Eq. (13). In the next section we further stress on the usage of these assumptions and on the consistency of the results obtained with our diagonalization procedure.

\section{SECOND ORDER CORRECTIONS}

In the previous section, after considering first order corrections to the diagonalization of the seesaw formula for neutrinos, it is found that the deviation of $\sin \theta_{12}$ from its TBM value is found to be undetermined. To know if this deviation can be determined in terms of model parameters, we study here the second order corrections to the diagonalization of the seesaw formula for neutrino masses. In order to do this we need to expand terms in $\frac{1}{\sqrt{\Delta m_{\text {atm }}^{2}}} m_{\nu}^{d}$ of Eq. (14) up to second order in $\epsilon_{i}, r, s, s_{13}$ and $\sqrt{\frac{\Delta m_{\text {sol }}^{2}}{\Delta m_{\text {atm }}^{2}}}$. Details related to this expansion and the analysis from that is explained below.

Expansion for $m_{\nu}^{s}$ and $U_{\mathrm{PMNS}}$, up to second order in $\epsilon_{i}, r$, $s$, and $s_{13}$ are given below

$$
\begin{gathered}
m_{\nu}^{s}=m_{\nu(0)}^{s}+m_{\nu(1)}^{s}+m_{\nu(2)}^{s}, \\
m_{\nu(2)}^{s}=\Delta m_{D} M_{R}^{-1}\left(\Delta m_{D}\right)^{T}, \\
U_{\mathrm{PMNS}}=U_{\mathrm{TBM}}+\Delta U+\Delta^{2} U,
\end{gathered}
$$

Here, the expressions for $m_{\nu(0)}^{s}, m_{\nu(1)}^{s}$, and $\Delta U$ can be found in Eqs. (16) and (18), while $U_{\mathrm{TBM}}$ can be seen in Eq. (6). After substituting the above described expansions for $m_{\nu}^{s}$ and $U_{\text {PMNS }}$ in Eq. (14), and also after using Eq. (13), $\frac{1}{\sqrt{\Delta m_{\text {amm }}^{2}}} m_{\nu}^{d}$ can be computed up to second order in $\epsilon_{i}, r, s, s_{13}$, and $\sqrt{\frac{\Delta m_{\text {sol }}^{2}}{\Delta m_{\mathrm{atm}}^{2}}}$. The full expressions for second order terms in $\frac{1}{\sqrt{\Delta m_{\text {atm }}^{2}}} m_{\nu}^{d}$ are given in Appendix A. Now, after using the results of Eq. (21) in Eq. (A1), the second order terms in $\frac{1}{\sqrt{\Delta m_{\mathrm{atm}}^{2}}} m_{\nu}^{d}$ will be simplified. These are given below.

$$
\begin{aligned}
& \frac{1}{\sqrt{\Delta m_{\mathrm{atm}}^{2}}} m_{\nu(2)}^{d}=\frac{1}{\sqrt{\Delta m_{\mathrm{atm}}^{2}}}\left(\begin{array}{lll}
x_{11}^{\prime \prime} & x_{12}^{\prime \prime} & x_{13}^{\prime \prime} \\
x_{12}^{\prime \prime} & x_{22}^{\prime \prime} & x_{23}^{\prime \prime} \\
x_{13}^{\prime \prime} & x_{23}^{\prime \prime} & x_{33}^{\prime \prime}
\end{array}\right), \\
& x_{11}^{\prime \prime}=0, \quad x_{12}^{\prime \prime}=\frac{a^{2}}{\sqrt{2} M_{\mathrm{sol}}}\left(2 \epsilon_{4}-\epsilon_{5}+\epsilon_{6}-3 r\right),
\end{aligned}
$$

Now, after equating the diagonal elements on both sides of Eq. (14), we get corrections up to second order to neutrino masses, which are given below. 


$$
\begin{aligned}
m_{1}= & 0, \quad m_{2}=\frac{3 a^{2}}{M_{\mathrm{sol}}}+\frac{2 a^{2}}{M_{\mathrm{sol}}}\left(\epsilon_{4}+\epsilon_{5}-\epsilon_{6}\right), \\
m_{3}= & \frac{2 e^{2}}{M_{\mathrm{atm}}}+\frac{4 e^{2}}{M_{\mathrm{atm}}}\left(\epsilon_{3}+s\right) \\
& +\frac{2 e^{2}}{M_{\mathrm{atm}}}\left(s_{13}^{2}+\epsilon_{3}^{2}+2 \epsilon_{3} s+2 s^{2}\right) .
\end{aligned}
$$

After demanding that the off-diagonal elements of $\frac{1}{\sqrt{\Delta m_{\text {atm }}^{2}}} m_{\nu}^{d}$ should be zero, we get the following three relations.

$$
\begin{gathered}
2 \epsilon_{4}-\epsilon_{5}+\epsilon_{6}=3 r, \\
s\left(3 s-2 \sqrt{2} e^{i \delta_{\mathrm{CP}}} s_{13}\right)+2 \epsilon_{3}\left(s-\sqrt{2} e^{i \delta_{\mathrm{CP}}} s_{13}\right)=0, \\
\sqrt{\frac{\Delta m_{\mathrm{sol}}^{2}}{\Delta m_{\mathrm{atm}}^{2}}} e^{i \phi}\left[\sqrt{2}\left(\epsilon_{5}+\epsilon_{6}+2 s\right)+2 e^{-i \delta_{\mathrm{CP}}} s_{13}\right] \\
-\left[2 \epsilon_{3}\left(\sqrt{2} s+e^{i \delta_{\mathrm{CP}}} s_{13}\right)+s\left(3 \sqrt{2} s+2 e^{i \delta_{\mathrm{CP}}} s_{13}\right)\right]=0 .
\end{gathered}
$$

While obtaining Eq. (29), we have used the expressions for $m_{2}$ and $m_{3}$ of Eq. (26). Here, $\phi$ is the Majorana phase difference in the neutrino masses $m_{2}$ and $m_{3}$.

From the expressions for neutrinos masses which are given in Eq. (26), we can see that the lightest neutrino mass is $m_{1}=0$. As already explained before, this result follows from the fact that there exists only two right-handed neutrinos in our model. But technically, this result will follow after using the relations of Eq. (21) in Eq. (A1). Since the relations in Eq. (21) are obtained after making the assumptions in Eq. (13), we can notice here on the consistency of the obtained results with our diagonalization procedure, which is described in the previous section. It is stated above that the lightest neutrino mass is zero in our model, and hence, only normal mass hierarchy is possible for neutrino masses. As a result of this the expressions for $m_{2}$ and $m_{3}$ of Eq. (26) can be fitted to $\sqrt{\Delta m_{\text {sol }}^{2}}$ and $\sqrt{\Delta m_{\mathrm{atm}}^{2}}$ respectively. While doing this fitting, we can notice that terms involving $\epsilon_{i}, s_{13}$ and $s$ give small corrections. Hence, we can see that $\frac{a^{2}}{M_{\mathrm{sol}}}$ and $\frac{e^{2}}{M_{\text {atm }}}$ can be of the order of $\sqrt{\Delta m_{\mathrm{sol}}^{2}}$ and $\sqrt{\Delta m_{\mathrm{atm}}^{2}}$ respectively. This result agrees with the assumption we have made in Eq. (13). Another point to be noticed here is that both the expressions for $m_{2}$ and $m_{3}$ depend on the complex $\epsilon_{i}$ parameters. As a result of this, both $m_{2}$ and $m_{3}$ can be complex. But since neutrino masses should be real, the complex phases in $m_{2}$ and $m_{3}$ can be absorbed into Majorana phases. Or else, another possibility is that we can choose the parameters $a$ and $e$ to be complex in such a way that $m_{2}$ and $m_{3}$ can be real. In this later case, the Majorana phases will become zero.

Regarding the neutrino mixing angles, we have explained in the previous section that the deviation in $\sin \theta_{12}$ from its TBM value is undetermined at the first order level corrections to diagonalization of the seesaw formula for neutrinos. But now after considering second order corrections, from Eq. (27) we can see that this deviation can be determined in terms of $\epsilon_{4}, \epsilon_{5}$, and $\epsilon_{6}$. In fact, out of these three $\epsilon$ parameters, only two can be determined by solving Eqs. (27)-(29). We can see that by solving Eq. (28), we can compute $\epsilon_{3}$ in terms of $s_{13}, s$, and $\delta_{C P}$. Now, by solving Eqs. (27) and (29), any two of the $\epsilon_{4}, \epsilon_{5}$, and $\epsilon_{6}$ can be found in terms of the neutrino masses and mixing angles. One among the $\epsilon_{4}, \epsilon_{5}$, and $\epsilon_{6}$ is still a free parameter, but it should be chosen to be small in order to be consistent with our analysis on neutrino mixing angles. After combining the results of Eqs. (21), (27)-(29), we can see that all the three neutrino mixing angles get deviations from their TBM values. Moreover, these deviations can be fitted to experimental values by choosing appropriate parametric space for $\epsilon_{i}$, which is the subject of the next section.

\section{NUMERICAL RESULTS}

We have explained how deviations from TBM pattern can be achieved by introducing the $\epsilon_{i}$ parameters in Eq. (7). Here, we numerically evaluate these parameters in order to be consistent with the neutrino oscillation data. In this regard, in our analysis, we have taken the best fit values for the two mass-squared differences among the neutrinos, which are given below [8].

$$
\begin{aligned}
\Delta m_{\mathrm{sol}}^{2} & =7.39 \times 10^{-5} \mathrm{eV}^{2}, \\
\Delta m_{\mathrm{atm}}^{2} & =2.525 \times 10^{-3} \mathrm{eV}^{2} .
\end{aligned}
$$

In the analysis, we have varied the three neutrino mixing angles and the $C P$ violating Dirac phase $\delta_{\mathrm{CP}}$ over the $3 \sigma$ ranges. These ranges are given below [8].

$\sin ^{2} \theta_{12}: 0.275 \rightarrow 0.350, \quad \sin ^{2} \theta_{23}: 0.418 \rightarrow 0.627$,

$\sin ^{2} \theta_{13}: 0.02045 \rightarrow 0.02439, \quad \delta_{C P}: 125^{\circ} \rightarrow 392^{\circ}$.

As described in the previous section, using the allowed values for neutrino oscillation observables, we can compute the $\epsilon_{i}$ parameters. Since these parameters are complex, we have resolved them in to real and imaginary parts, whose expressions are given below.

$$
\epsilon_{i}=\operatorname{Re}\left(\epsilon_{i}\right)+i \operatorname{Im}\left(\epsilon_{i}\right)
$$

In order to be compatible with the above mentioned neutrino oscillation observables, we have obtained the allowed ranges for $\operatorname{Re}\left(\epsilon_{i}\right)$ and $\operatorname{Im}\left(\epsilon_{i}\right)$. These results are given in Table I. In this table, the allowed ranges for real and imaginary parts of $\epsilon_{5}$ and $\epsilon_{6}$ are obtained by fixing the values for $\epsilon_{4}$ and also by taking the phase $\phi=0$. Moreover, in this table, the allowed ranges for $\operatorname{Im}\left(\epsilon_{2}\right), \operatorname{Im}\left(\epsilon_{3}\right)$ are 
TABLE I. Allowed ranges for the real and imaginary parts of the $\epsilon_{i}$ parameters. For details, see the text.

\begin{tabular}{lccccc}
\hline \hline $\operatorname{Re}\left(\epsilon_{1}\right)$ & $\operatorname{Im}\left(\epsilon_{1}\right)$ & $\operatorname{Re}\left(\epsilon_{2}\right)$ & $\operatorname{Im}\left(\epsilon_{2}\right), \operatorname{Im}\left(\epsilon_{3}\right)$ & $\operatorname{Re}\left(\epsilon_{3}\right)$ \\
\hline$(-0.221,0.221)$ & $(-0.221,0.182)$ & $(-0.106,0.225)$ & $(-0.064,0.064)$ & $(-0.15,0.095)$ \\
\hline \hline$\phi$ & $\epsilon_{4}$ & $\operatorname{Re}\left(\epsilon_{5}\right)$ & $\operatorname{Im}\left(\epsilon_{5}\right)$ & $\operatorname{Re}\left(\epsilon_{6}\right)$ & $\operatorname{Im}\left(\epsilon_{6}\right)$ \\
\hline 0 & 0.1 & $(-0.084,0.462)$ & $(-0.119,0.101)$ & $(-0.375,0.168)$ & $(-0.119,0.101)$ \\
0 & -0.1 & $(-0.282,0.26)$ & $(-0.119,0.101)$ & $(-0.175,0.367)$ & $(-0.119,0.101)$ \\
0 & $0.1 i$ & $(-0.182,0.362)$ & $(-0.019,0.199)$ & $(-0.275,0.267)$ & $(-0.219,0.001)$ \\
0 & $-0.1 i$ & $(-0.182,0.362)$ & $(-0.219,0.001)$ & $(-0.275,0.267)$ & $(-0.019,0.199)$ \\
\hline \hline
\end{tabular}

same. This result follows from Eq. (21), which implies $\operatorname{Im}\left(\epsilon_{2}\right)=\operatorname{Im}\left(\epsilon_{3}\right)$. From the results given in Table I, we notice that except for $\operatorname{Re}\left(\epsilon_{5}\right)$ and $\operatorname{Re}\left(\epsilon_{6}\right)$, the magnitude of other parameters are less than about $\sqrt{2} s_{13} \sim 0.221$. The magnitudes of $\operatorname{Re}\left(\epsilon_{5}\right)$ and $\operatorname{Re}\left(\epsilon_{6}\right)$ can become as large as 0.46 and 0.37 respectively, for the case of $\phi=0$ and $\epsilon_{4}=0.1$. We have varied $\phi$ away from zero, by fixing $\epsilon_{4}=0.1$, and have computed real and imaginary parts of $\epsilon_{5}$ and $\epsilon_{6}$. In these cases, we have found that the maximum values for $\left|\operatorname{Re}\left(\epsilon_{5}\right)\right|$ and $\left|\operatorname{Re}\left(\epsilon_{6}\right)\right|$ to be lying between about 0.37 and 0.63 , whereas, the maximum values for $\left|\operatorname{Im}\left(\epsilon_{5}\right)\right|$ and $\left|\operatorname{Im}\left(\epsilon_{6}\right)\right|$ are found to be less than 0.2.

We have explained previously that the diagonalization of the seesaw formula of our model is done by assuming that the magnitudes of real and imaginary parts of $\epsilon_{i}$ to be less than or of the order of $\sqrt{\frac{\Delta m_{\text {sol }}^{2}}{\Delta m_{\text {atm }}^{2}}} \sim \sin \theta_{13}$. From the numerical results presented above, we can notice that, real and imaginary parts of $\epsilon_{i}$ satisfy the above mentioned assumption, except for $\operatorname{Re}\left(\epsilon_{5}\right)$ and $\operatorname{Re}\left(\epsilon_{6}\right)$. The maximum values for $\left|\operatorname{Re}\left(\epsilon_{5}\right)\right|$ and $\left|\operatorname{Re}\left(\epsilon_{6}\right)\right|$ can be about 0.4, depending on $\epsilon_{4}$ and $\phi$ values. At these maximum values, the analytic expressions presented in previous sections may not give accurate results, since square of 0.4 is not negligible in comparison to unity. One can notice that $\epsilon_{5}$ and $\epsilon_{6}$ contribute linearly to neutrino oscillation observables in the second order corrections of our analysis. As a result of this, corrections at the third order level to the above mentioned observables are not negligible around these maximum values. On the other hand, one would like to know, if by restricting the values of $\left|\operatorname{Re}\left(\epsilon_{5}\right)\right|$ and $\left|\operatorname{Re}\left(\epsilon_{6}\right)\right|$ to be small, the analytic expressions of previous sections are sufficient enough to give accurate numerical results. For this reason, we have computed allowed values for neutrino mixing angles and $\delta_{C P}$ by demanding $\left|\operatorname{Re}\left(\epsilon_{5}\right)\right|$ and $\left|\operatorname{Re}\left(\epsilon_{6}\right)\right|$ to be less than 0.23 for the case of $\phi=0$ and $\epsilon_{4}=0.1$. These results are given in Figure 1. Now, after combining the results of Table I, one can notice that in the allowed regions of Fig. 1, the magnitudes of real and imaginary parts of all $\epsilon_{i}$ are less than 0.23 , which is about $\sqrt{2} s_{13}$. Hence, in the allowed regions of Figure $1, \epsilon_{i}$ satisfy the assumptions we have made in order to diagonalize the seesaw formula of our model.
We have discretized the axes of $s_{13}^{2}, s_{23}^{2}, s_{12}^{2}, \delta_{C P}$ in order to obtain the results in Figure 1. From this figure, one can notice that the allowed range for $s_{13}^{2}$ is almost unconstrained. However, the allowed regions for $s_{23}^{2}$ and $s_{12}^{2}$ are constrained for some specific values of $\delta_{C P}$. A notable feature from Fig. 1 is that points with $s_{12}^{2}=0.275$ are excluded for any value of $\delta_{C P}$. Although the results in Figure 1 are obtained for $\phi=0$ and $\epsilon_{4}=0.1$, a similar analysis can be done for any other values of $\phi$ and $\epsilon_{4}$. Hence, in our work, we can find a region where $\epsilon_{i}$ parameters are small and consistently explain the deviation from TBM pattern in the neutrino sector.

Recently, results from global fits to neutrino oscillation data have been updated in Ref. [17]. These results prefer normal hierarchy for neutrino masses. In our framework, which is based on CSD, the analytic results from previous section show that neutrino masses have normal hierarchy. Hence, the prediction of our CSD scenario is favorable by the recent neutrino oscillation data. In the case of normal mass hierarchy, recent results from neutrino oscillation data prefer second octant for $\sin ^{2} \theta_{23}$ with the best fit value of 0.566 [17]. The $C P$ violating phase $\delta_{\mathrm{CP}}$, in the above case, has a best fit value of $1.2 \pi$ [17]. From the results given in Fig. 1, we notice lower octant values for $\sin ^{2} \theta_{23}$ are excluded for $\delta_{C P}$ around $\pi$. Hence, the numerical results of our work are compatible with the recent neutrino oscillation data.

\section{A MODEL FOR OUR DIRAC MASS MATRIX}

In this section, we construct a model in order to justify the structure of our Dirac mass matrix of Eq. (7) and also explain the small values for $\epsilon_{i}$. For this purpose, we introduce a flavor symmetry $S U(3)$ and also the following scalar fields: $\phi_{a}, \phi_{s}, \phi_{a}^{\prime}, \phi_{s}^{\prime}$. These scalar fields are singlets under the standard model gauge group, but otherwise, charged under the $S U(3)$. The lepton doublets $L$, where we have suppressed generation index, are charged under this flavor symmetry. The Higgs doublet $H$ and the two righthanded neutrinos $\nu_{R}^{\text {atm }}, \nu_{R}^{\text {sol }}$ are singlets under $S U(3)$. To get the masses for right-handed neutrinos, we introduce the following additional scalar fields, which are standard model gauge singlets: $\chi_{a}, \chi_{s}$. To explain the smallness of $\epsilon_{i}$ 

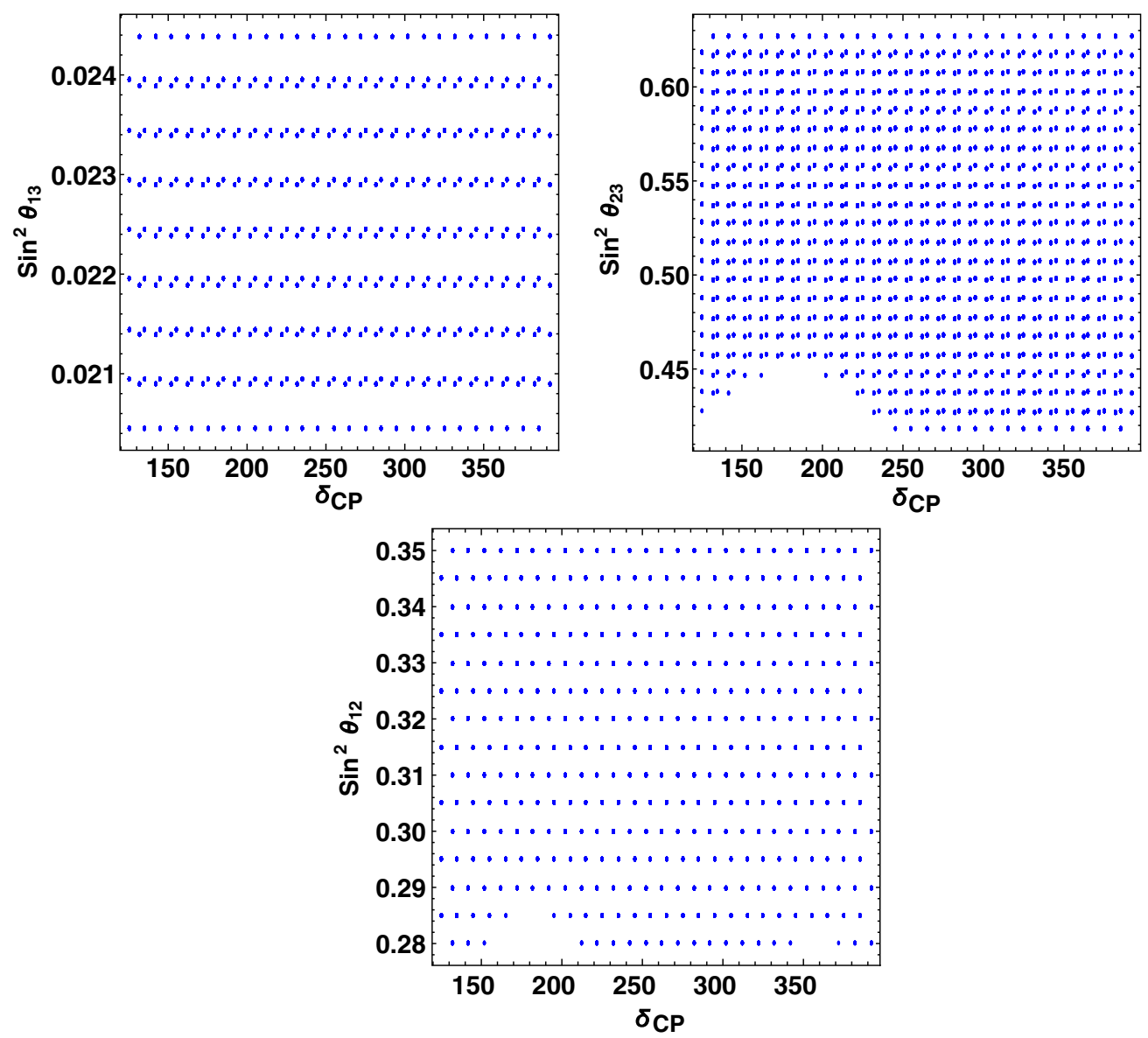

FIG. 1. Allowed regions in neutrino mixing angles and $\delta_{\mathrm{CP}}$ by demanding $\left|\operatorname{Re}\left(\epsilon_{5}\right)\right|$ and $\left|\operatorname{Re}\left(\epsilon_{6}\right)\right|$ to be less than 0.23 , for the case of $\phi=0$ and $\epsilon_{4}=0.1 . \delta_{\mathrm{CP}}$ is expressed in degrees.

parameters, we propose the scalar field $\xi$ which is a standard model gauge singlet. To forbid unwanted interactions in our model we introduce a discreet symmetry $Z_{3} \times Z_{3}^{\prime}$. In Table II, charges assignments of the fields, which are relevant to neutrino sector, are given. With these charge assignments, the leading terms in the Lagrangian are

$$
\begin{aligned}
\mathcal{L}= & \frac{\phi_{a}}{M_{P}} \bar{L} \nu_{R}^{\mathrm{atm}} H+\frac{\phi_{s}}{M_{P}} \bar{L} \nu_{R}^{\mathrm{sol}} H \\
& +\frac{\xi}{M_{P}} \frac{\phi_{a}^{\prime}}{M_{P}} \bar{L} \nu_{R}^{\mathrm{atm}} H+\frac{\xi}{M_{P}} \frac{\phi_{s}^{\prime}}{M_{P}} \bar{L} \nu_{R}^{\mathrm{sol}} H \\
& +\frac{\chi_{a}}{2} \overline{\left(\nu_{R}^{\mathrm{atm}}\right)^{c}} \nu_{R}^{\mathrm{atm}}+\frac{\chi_{s}}{2} \overline{\left(\nu_{R}^{\mathrm{sol}}\right)^{c}} \nu_{R}^{\mathrm{sol}}+\text { H.c. }
\end{aligned}
$$

TABLE II. Charge assignments of the relevant fields under the flavor symmetry $S U(3) \times Z_{3} \times Z_{3}^{\prime}$ are given. Here, $\omega=e^{2 \pi i / 3}$. For other details, see the text.

\begin{tabular}{lccccccccccc}
\hline \hline & $\phi_{a}$ & $\phi_{s}$ & $\phi_{a}^{\prime}$ & $\phi_{s}^{\prime}$ & $\xi$ & $\chi_{a}$ & $\chi_{s}$ & $\nu_{R}^{\text {atm }}$ & $\nu_{R}^{\text {sol }}$ & $L$ & $H$ \\
\hline$S U(3)$ & 3 & 3 & 3 & 3 & 1 & 1 & 1 & 1 & 1 & 3 & 1 \\
$Z_{3}$ & $\omega$ & $\omega^{2}$ & $\omega$ & $\omega^{2}$ & 1 & $\omega^{2}$ & $\omega$ & $\omega^{2}$ & $\omega$ & 1 & 1 \\
$Z_{3}^{\prime}$ & $\omega^{2}$ & $\omega^{2}$ & $\omega$ & $\omega$ & $\omega$ & $\omega$ & $\omega$ & $\omega$ & $\omega$ & 1 & 1 \\
\hline \hline
\end{tabular}

Here, $M_{P} \sim 2 \times 10^{18} \mathrm{GeV}$ is the reduced Planck scale, which is the cutoff scale for this model. The reason for choosing the Planck scale as the cutoff of the model is explained later this section.

The first four terms of Eq. (33) generate effective Yukawa couplings for neutrinos after the following scalar fields acquire vevs: $\phi_{a}, \phi_{s}, \phi_{a}^{\prime}, \phi_{s}^{\prime}, \xi$. The vevs of $\phi_{a}, \phi_{s}$ give leading contribution to effective Yukawa couplings. In order to explain the structure of Dirac mass matrix of Eq. (7) we assume that these vevs to have the following pattern

$$
\frac{\left\langle\phi_{a}\right\rangle}{M_{P}}=y_{a}\left(\begin{array}{l}
0 \\
1 \\
1
\end{array}\right), \quad \frac{\left\langle\phi_{s}\right\rangle}{M_{P}}=y_{s}\left(\begin{array}{c}
1 \\
1 \\
-1
\end{array}\right)
$$

Here, $y_{a}, y_{s}$ are dimensionless quantities. The above pattern of vevs can be obtained by tuning parameters in the scalar potential of this model, which is described in the next section. It is to be noted that the problem related to vev pattern of Eq. (34) has been addressed in Ref. [6]. The vevs of $\phi_{a}^{\prime}, \phi_{s}^{\prime}, \xi$ give subleading contribution to Yukawa couplings for neutrinos. Here, we need not assume any pattern 
for the vevs of $\phi_{a}^{\prime}, \phi_{s}^{\prime}$. Hence, after writing $\frac{\langle\xi\rangle}{M_{P}}=\epsilon$, we can have

$$
\begin{gathered}
\frac{\langle\xi\rangle}{M_{P}} \frac{\left\langle\phi_{a}^{\prime}\right\rangle}{M_{P}}=y_{a}\left(\begin{array}{l}
y_{1} \\
y_{2} \\
y_{3}
\end{array}\right) \epsilon=y_{a}\left(\begin{array}{c}
\epsilon_{1} \\
\epsilon_{2} \\
\epsilon_{3}
\end{array}\right), \\
\frac{\langle\xi\rangle}{M_{P}} \frac{\left\langle\phi_{s}^{\prime}\right\rangle}{M_{P}}=y_{s}\left(\begin{array}{l}
y_{1}^{\prime} \\
y_{2}^{\prime} \\
y_{3}^{\prime}
\end{array}\right) \epsilon=y_{s}\left(\begin{array}{c}
\epsilon_{4} \\
\epsilon_{5} \\
\epsilon_{6}
\end{array}\right) .
\end{gathered}
$$

Here, $y_{i}, y_{i}^{\prime}$, where $i=1, \ldots, 3$, are $\mathcal{O}(1)$ parameters. By taking $\frac{\langle\xi\rangle}{M_{P}}=\epsilon \sim 0.1$, we get all $\epsilon_{i}$ to be around 0.1 . We can see that the smallness of $\epsilon_{i}$ parameters can be explained if the field $\xi$ acquire vev around one order less than the $M_{P}$. Now, using Eqs. (34) and (35) in Eq. (33), after electroweak symmetry breaking, we get the structure of Dirac mass matrix which is proposed in Eq. (7). Finally, the last two terms of Eq. (33) give diagonal masses to right-handed neutrinos, after the fields $\chi_{a}, \chi_{s}$ acquire vevs.

In the above, by proposing a model, we have explained the mass structures of Dirac and right-handed neutrinos of this work. In order to explain these mass structures, the extra scalar fields proposed in this model need to acquire vevs and thereby break the flavor symmetry $S U(3) \times Z_{3} \times Z_{3}^{\prime}$ spontaneously. Here we quantify the scales of these vevs. It is stated above that $\left\langle\chi_{a}\right\rangle$ and $\left\langle\chi_{s}\right\rangle$ generate masses for right-handed neutrinos. Requiring that these masses to be around $1 \mathrm{TeV}$, we should have: $\left\langle\chi_{a}\right\rangle,\left\langle\chi_{s}\right\rangle \sim 1 \mathrm{TeV}$. One motivation for choosing $\mathrm{TeV}$ scale masses for right-handed neutrinos is that they can be detected in the LHC experiment. Another motivation for choosing the above mass scale for right-handed neutrinos is shortly explained below. The vev of $\xi$ can be found from the fact that it explains the smallness of $\epsilon_{i}$ parameters. In order to explain this smallness, we have described above that we need to have $\frac{\langle\xi\rangle}{M_{P}} \sim 0.1$. From this we get $\langle\xi\rangle \sim 10^{17} \mathrm{GeV}$. Finally, the vevs of $\phi_{a}, \phi_{s}, \phi_{a}^{\prime}, \phi_{s}^{\prime}$ can be determined from the reasoning that they generate effective Yukawa couplings for neutrinos. Since we have taken right-handed neutrino masses to be around $1 \mathrm{TeV}$, from seesaw formula for active neutrinos, we can estimate the magnitude of Yukawa couplings for neutrinos by having the active neutrino masses to be of $\mathcal{O}(0.1) \mathrm{eV}$. From this estimation, we have found that $\left\langle\phi_{a}\right\rangle,\left\langle\phi_{s}\right\rangle,\left\langle\phi_{a}^{\prime}\right\rangle,\left\langle\phi_{s}^{\prime}\right\rangle \sim 10^{12} \mathrm{GeV}$. After finding the vevs of the additional scalar fields of this model, we notice that there is a large hierarchy among these vevs. We can achieve this hierarchy, in this model, by appropriately fixing the relevant parameters in the scalar potential among the above mentioned scalar fields. Analysis related to the scalar potential of our model is presented in Appendix B.

It is stated that $M_{P}$ is the cutoff scale for the above described model. One can notice that the hierarchy between $\langle\xi\rangle$ and $M_{P}$ is very less. However, the hierarchy in the vevs of other scalar fields such as $\phi_{a}, \phi_{s}, \phi_{a}^{\prime}, \phi_{s}^{\prime}, \chi_{a}, \chi_{s}$ is very large with respect to $M_{P}$. Here, we explain this hierarchy by motivating the above described model from supersymmetry [18]. Since supersymmetry is not exact symmetry, one possibility is to break supersymmetry spontaneously by hidden sector fields and the effects of this breaking are mediated to visible sector via gravity mediated interactions [18]. Models based on this mechanism are known as supergravity models, where hidden sector fields can interact with visible sector fields with Planck scale suppressed nonrenormalizable terms. In these models, hidden sector fields can acquire vevs around the intermediate scale of $\Lambda \sim 10^{11} \mathrm{GeV}$ and the $\mathrm{TeV}$ scale can be generated by $\frac{\Lambda^{2}}{M_{P}}$. Based on this, models have been proposed in order to conceive TeV scale masses for right-handed neutrinos [19]. In our model, which is described above, the vevs for $\chi_{a}, \chi_{s}$ are around $\mathrm{TeV}$ and the vevs for $\phi_{a}, \phi_{s}, \phi_{a}^{\prime}, \phi_{s}^{\prime}$ are close to the intermediate scale. Hence, we can explain the hierarchy in the vev of these fields by embedding our model in a supergravity setup. In fact, for this reason we have chosen $M_{P}$ as the cutoff scale to our model.

Above, we have motivated the hierarchy in the vevs of scalar fields of our model from a supergravity setup. On the other hand, in order to achieve this hierarchy in the current framework, we have carried out an analysis on the scalar potential of our model in Appendix B. In this appendix, we have given the invariant scalar potential under the flavor symmetry $S U(3) \times Z_{3} \times Z_{3}^{\prime}$. After minimizing this scalar potential, the vevs of various scalar fields of our model have been determined. It is shown in Appendix B that by tuning the parameters of the scalar potential, the required hierarchy among the vevs of the scalar fields can be achieved. It has been argued that the desired vacuum alignment for $\phi_{a}$ and $\phi_{s}$ can be achieved by tuning the necessary parameters in the scalar potential. Although the scalar potential in Appendix $\mathrm{B}$ is at tree level, due to large number of parameters, the above mentioned vacuum alignment is still possible even after including the radiative corrections to the scalar potential. It is described in Appendix B that in order to achieve the desired hierarchy in the vevs of scalar fields, some couplings in the scalar potential should be suppressed to as low as $10^{-32}$. To explain the smallness of these couplings, one can extend the flavor symmetry of our model to $S U(3) \times Z_{3} \times Z_{3}^{\prime} \times Z_{2} \times Z_{2}^{\prime}$. We have noticed that under the additional symmetry $Z_{2} \times Z_{2}^{\prime}$, charge assignments for fields can be done in such a way that terms in Eq. (33) are allowed but the terms in the scalar potential with couplings of the order of $10^{-32}$ are forbidden. After doing that, one can motivate the smallness of these couplings as a soft breaking of the additional flavor symmetry $Z_{2} \times Z_{2}^{\prime}$. To explain the smallness of other couplings in the scalar potential, either one can extend the above flavor symmetry or one needs to device a new mechanism. 
It is described previously that apart from the Higgs field, rest of the scalar fields are charged under the flavor symmetry $S U(3) \times Z_{3} \times Z_{3}^{\prime}$. This symmetry is spontaneously broken by the vevs of the scalar fields. Since these fields are complex, apart from the Higgs boson, a total of thirty real scalar fields exist in our model. By choosing the flavor symmetry $S U(3)$ to be gauged, after spontaneous symmetry breaking, apart from the Higgs boson, twenty two real scalar fields remain in our model. All these fields have mixing masses. We estimate the masses for these fields to be around the scales at which they acquire vevs. The gauge bosons of the flavor symmetry $S U(3)$ can get masses around $10^{12} \mathrm{GeV}$. Since lepton doublets are charged under the flavor $S U(3)$, the above gauge bosons have couplings to leptons. A study on the phenomenology of the additional fields of our model is out of the scope of this work.

\section{CONCLUSIONS}

In this work, we have attempted to explain the neutrino mixing in order to be consistent with the current neutrino oscillation data. From the current data, it is known that $\theta_{13} \neq 0$, and hence, the neutrino mixing angles deviate away from the TBM pattern. Earlier, to explain the TBM pattern in neutrino sector, CSD model has been proposed. Here, we have considered a phenomenological model, where we have modified the neutrino Yukawa couplings of CSD model, by introducing small $\epsilon_{i}$ parameters which are complex. We have assumed real and imaginary parts of $\epsilon_{i}$ to be less than or of the order of $\sin \theta_{13} \sim \sqrt{\frac{\Delta m_{\text {sol }}^{2}}{\Delta m_{\text {atm }}^{2}}}$. Thereafter, we have followed an approximation procedure in order to diagonalize the seesaw formula for light neutrinos in our model. We have computed expressions, up to second order level, to neutrino masses and mixing angles in terms of small $\epsilon_{i}$ parameters. Using these expressions we have demonstrated that neutrino mixing angles can deviate away from their TBM values by appropriately choosing the $\epsilon_{i}$ values. Finally, we have constructed a model in order to justify the neutrino Yukawa coupling structure of our phenomenological model.

\section{APPENDIX A: FULL EXPRESSIONS AT SECOND ORDER CORRECTIONS}

The full form of second order terms in $\frac{1}{\sqrt{\Delta m_{\text {atm }}^{2}}} m_{\nu}^{d}$ are given below.

$$
\begin{aligned}
& \frac{1}{\sqrt{\Delta m_{\mathrm{atm}}^{2}}} m_{\nu(2)}^{d}=\frac{1}{\sqrt{\Delta m_{\mathrm{atm}}^{2}}}\left(\begin{array}{ccc}
x_{11}^{\prime \prime} & x_{12}^{\prime \prime} & x_{13}^{\prime \prime} \\
x_{12}^{\prime \prime} & x_{22}^{\prime \prime} & x_{23}^{\prime \prime} \\
x_{13}^{\prime \prime} & x_{23}^{\prime \prime} & x_{33}^{\prime \prime}
\end{array}\right), \\
& x_{11}^{\prime \prime}=\frac{e^{2}}{6 M_{\mathrm{atm}}}\left[4 \epsilon_{1}^{2}+\epsilon_{2}^{2}+\left(\epsilon_{3}+2 s\right)^{2}-4 \sqrt{2} e^{i \delta_{C P}} s_{13}\left(\epsilon_{3}+2 s\right)+8 e^{2 i \delta_{C P}} s_{13}^{2}\right. \\
& \left.-2 \epsilon_{2}\left(\epsilon_{3}+2 s-2 \sqrt{2} e^{i \delta_{C P}} s_{13}\right)-4 \epsilon_{1}\left(\epsilon_{2}-\epsilon_{3}-2 s+2 \sqrt{2} e^{i \delta_{C P}} s_{13}\right)\right], \\
& x_{12}^{\prime \prime}=\frac{a^{2}}{\sqrt{2} M_{\mathrm{sol}}}\left(2 \epsilon_{4}-\epsilon_{5}+\epsilon_{6}-3 r\right)+\frac{e^{2}}{6 M_{\mathrm{atm}}}\left[\sqrt{2}\left(2 \epsilon_{1}^{2}-\epsilon_{2}^{2}\right)-\sqrt{2}\left(\epsilon_{3}+2 s\right)^{2}\right. \\
& \left.+2 e^{i \delta_{C P}} s_{13}\left(\epsilon_{3}+2 s\right)+4 \sqrt{2} e^{2 i \delta_{C P}} s_{13}^{2}+\epsilon_{1}\left(\sqrt{2}\left(\epsilon_{2}-\epsilon_{3}-2 s\right)-8 e^{i \delta_{C P}} s_{13}\right)+2 \epsilon_{2}\left(\sqrt{2}\left(\epsilon_{3}+2 s\right)-e^{i \delta_{C P}} s_{13}\right)\right], \\
& x_{13}^{\prime \prime}=\frac{e^{2}}{\sqrt{2} M_{\mathrm{sol}}}\left[-\epsilon_{2}^{2}+\epsilon_{3}^{2}+2 \epsilon_{1}\left(\epsilon_{2}+\epsilon_{3}-r\right)+2 \epsilon_{3} r+4 \epsilon_{3} s+4 r s+2 s^{2}-4 \sqrt{2} e^{i \delta_{C P}} s_{13} \epsilon_{3}\right. \\
& \left.+2 \sqrt{2} e^{i \delta_{C P}} s_{13} r-2 \epsilon_{2}\left(r-2 s+2 \sqrt{2} e^{i \delta_{C P}} s_{13}\right)\right], \\
& x_{22}^{\prime \prime}=\frac{2 a^{2}}{M_{\mathrm{sol}}}\left(\epsilon_{4}+\epsilon_{5}-\epsilon_{6}\right)+\frac{e^{2}}{3 M_{\mathrm{atm}}}\left[\epsilon_{1}^{2}+\epsilon_{2}^{2}+\left(\epsilon_{3}+2 s\right)^{2}+2 \sqrt{2} e^{i \delta_{C P}} s_{13}\left(\epsilon_{3}+2 s\right)\right. \\
& \left.+2 e^{2 i \delta_{C P}} s_{13}^{2}+2 \epsilon_{1}\left(\epsilon_{2}-\epsilon_{3}-2 s-\sqrt{2} e^{i \delta_{C P}} s_{13}\right)-2 \epsilon_{2}\left(\epsilon_{3}+2 s+\sqrt{2} e^{i \delta_{C P}} s_{13}\right)\right], \\
& x_{23}^{\prime \prime}=\frac{\sqrt{3} a^{2}}{2 M_{\mathrm{sol}}}\left[\sqrt{2}\left(\epsilon_{5}+\epsilon_{6}+2 s\right)+2 e^{-i \delta_{C P}} s_{13}\right]+\frac{e^{2}}{2 \sqrt{3} M_{\mathrm{atm}}}\left[\sqrt{2}\left(\epsilon_{2}^{2}-\epsilon_{3}^{2}+\epsilon_{3} r\right)\right. \\
& \left.+\sqrt{2} \epsilon_{1}\left(\epsilon_{2}+\epsilon_{3}+2 r\right)-\sqrt{2}\left(4 \epsilon_{3} s-2 r s+2 s^{2}\right)-4 e^{i \delta_{C P}} s_{13}\left(\epsilon_{3}+r\right)-\epsilon_{2}\left(\sqrt{2} r+4 \sqrt{2} s+4 e^{i \delta_{C P}} s_{13}\right)\right], \\
& x_{33}^{\prime \prime}=\frac{e^{2}}{2 M_{\mathrm{atm}}}\left[4 \sqrt{2} \epsilon_{1} s_{13} e^{-i \delta_{C P}}+\epsilon_{2}^{2}+\epsilon_{3}^{2}-4 \epsilon_{3} s+2 \epsilon_{2}\left(\epsilon_{3}+2 s\right)-4\left(s^{2}+s_{13}^{2}\right)\right] \text {. }
\end{aligned}
$$




\section{APPENDIX B: ANALYSIS OF SCALAR POTENTIAL}

Here we analyze the scalar potential of the model, which is described in Sec. VI. The invariant scalar potential of this model is given below.

$$
\begin{aligned}
& V=m_{\phi_{a}}^{2} \boldsymbol{\phi}_{a}^{\dagger} \boldsymbol{\phi}_{a}+m_{\phi_{s}}^{2} \boldsymbol{\phi}_{s}^{\dagger} \boldsymbol{\phi}_{s}+m_{\phi_{a}^{\prime}}^{2} \boldsymbol{\phi}_{a}^{\prime \dagger} \phi_{a}^{\prime}+m_{\phi_{s}^{\prime}}^{2} \boldsymbol{\phi}_{s}^{\prime \dagger} \boldsymbol{\phi}_{s}^{\prime}+m_{\chi_{a}}^{2} \chi_{a}^{*} \chi_{a}+m_{\chi_{s}}^{2} \chi_{s}^{*} \chi_{s}+m_{\xi}^{2} \xi^{*} \xi \\
& +m_{H}^{2} H^{\dagger} H+\lambda_{\phi_{a}}\left(\phi_{a}^{\dagger} \phi_{a}\right)^{2}+\lambda_{\phi_{s}}\left(\phi_{s}^{\dagger} \phi_{s}\right)^{2}+\lambda_{\phi_{a}^{\prime}}\left(\phi_{a}^{\prime \dagger} \phi_{a}^{\prime}\right)^{2}+\lambda_{\phi_{s}^{\prime}}\left(\phi_{s}^{\prime \dagger} \phi_{s}^{\prime}\right)^{2}+\lambda_{\chi_{a}}\left(\chi_{a}^{*} \chi_{a}\right)^{2} \\
& +\lambda_{\chi_{s}}\left(\chi_{s}^{*} \chi_{s}\right)^{2}+\lambda_{\xi}\left(\xi^{*} \xi\right)^{2}+\lambda_{H}\left(H^{\dagger} H\right)^{2}+\lambda_{\phi_{a} H}\left(\phi_{a}^{\dagger} \phi_{a}\right)\left(H^{\dagger} H\right)+\lambda_{\phi_{s} H}\left(\phi_{s}^{\dagger} \phi_{s}\right)\left(H^{\dagger} H\right) \\
& +\lambda_{\phi_{a}^{\prime} H}\left(\phi_{a}^{\prime \dagger} \phi_{a}^{\prime}\right)\left(H^{\dagger} H\right)+\lambda_{\phi_{s}^{\prime} H}\left(\phi_{s}^{\prime \dagger} \phi_{s}^{\prime}\right)\left(H^{\dagger} H\right)+\lambda_{\chi_{a} H}\left(\chi_{a}^{*} \chi_{a}\right)\left(H^{\dagger} H\right)+\lambda_{\chi_{s} H}\left(\chi_{s}^{*} \chi_{s}\right)\left(H^{\dagger} H\right) \\
& +\lambda_{\xi H}\left(\xi^{*} \xi\right)\left(H^{\dagger} H\right)+\left[a_{1}\left(\phi_{a}^{\dagger} \phi_{a}^{\prime}\right) \xi+a_{2}\left(\phi_{a}^{\dagger} \phi_{s}^{\prime}\right) \chi_{a}+a_{3}\left(\phi_{s}^{\dagger} \phi_{a}^{\prime}\right) \chi_{s}+a_{4}\left(\phi_{s}^{\dagger} \phi_{s}^{\prime}\right) \xi+a_{5} \chi_{a} \chi_{s} \xi\right. \\
& \left.+a_{6} \xi^{3}+a_{7} \chi_{a}^{3}+a_{8} \chi_{s}^{3}+\text { H.c. }\right]+b_{1}\left(\phi_{a}^{\dagger} \phi_{a}\right)\left(\phi_{s}^{\dagger} \phi_{s}\right)+b_{2}\left(\phi_{a}^{\dagger} \phi_{a}\right)\left(\phi_{a}^{\prime \dagger} \phi_{a}^{\prime}\right)+b_{3}\left(\phi_{a}^{\dagger} \phi_{a}\right)\left(\phi_{s}^{\prime \dagger} \phi_{s}^{\prime}\right) \\
& +b_{4}\left(\phi_{s}^{\dagger} \phi_{s}\right)\left(\phi_{a}^{\prime \dagger} \phi_{a}^{\prime}\right)+b_{5}\left(\phi_{s}^{\dagger} \phi_{s}\right)\left(\phi_{s}^{\prime \dagger} \phi_{s}^{\prime}\right)+b_{6}\left(\phi_{a}^{\prime \dagger} \phi_{a}^{\prime}\right)\left(\phi_{s}^{\prime \dagger} \phi_{s}^{\prime}\right)+b_{7}\left(\phi_{a}^{\dagger} \phi_{s}^{\prime}\right)\left(\phi_{s}^{\prime \dagger} \phi_{a}\right) \\
& +b_{8}\left(\phi_{a}^{\dagger} \phi_{s}\right)\left(\phi_{s}^{\dagger} \phi_{a}\right)+\left[b_{9}\left(\phi_{s}^{\dagger} \phi_{a}\right)\left(\phi_{a}^{\prime \dagger} \phi_{s}^{\prime}\right)+b_{10}\left(\phi_{s}^{\dagger} \boldsymbol{\phi}_{s}^{\prime}\right)\left(\phi_{a}^{\prime \dagger} \phi_{a}\right)+\text { H.c. }\right]+b_{11}\left(\phi_{s}^{\dagger} \boldsymbol{\phi}_{a}^{\prime}\right)\left(\phi_{a}^{\prime \dagger} \phi_{s}\right) \\
& +b_{12}\left(\phi_{s}^{\prime \dagger} \phi_{s}\right)\left(\phi_{s}^{\dagger} \phi_{s}^{\prime}\right)+b_{13}\left(\phi_{s}^{\prime \dagger} \phi_{a}^{\prime}\right)\left(\phi_{a}^{\prime \dagger} \phi_{s}^{\prime}\right)+b_{14}\left(\phi_{a}^{\dagger} \phi_{a}^{\prime}\right)\left(\phi_{a}^{\prime \dagger} \phi_{a}\right)+c_{1}\left(\phi_{a}^{\dagger} \phi_{a}\right) \xi^{*} \xi+c_{2}\left(\phi_{s}^{\dagger} \phi_{s}\right) \xi^{*} \xi \\
& +c_{3}\left(\phi_{a}^{\prime \dagger} \phi_{a}^{\prime}\right) \xi^{*} \xi+c_{4}\left(\phi_{s}^{\prime \dagger} \phi_{s}^{\prime}\right) \xi^{*} \xi+c_{5}\left(\phi_{a}^{\dagger} \phi_{a}\right) \chi_{a}^{*} \chi_{a}+c_{6}\left(\phi_{s}^{\dagger} \phi_{s}\right) \chi_{a}^{*} \chi_{a}+c_{7}\left(\phi_{a}^{\prime \dagger} \phi_{a}^{\prime}\right) \chi_{a}^{*} \chi_{a} \\
& +c_{8}\left(\phi_{s}^{\prime \dagger} \phi_{s}^{\prime}\right) \chi_{a}^{*} \chi_{a}+c_{9}\left(\phi_{a}^{\dagger} \phi_{a}\right) \chi_{s}^{*} \chi_{s}+c_{10}\left(\phi_{s}^{\dagger} \phi_{s}\right) \chi_{s}^{*} \chi_{s}+c_{11}\left(\phi_{a}^{\prime \dagger} \phi_{a}^{\prime}\right) \chi_{s}^{*} \chi_{s}+c_{12}\left(\phi_{s}^{\prime \dagger} \phi_{s}^{\prime}\right) \chi_{s}^{*} \chi_{s} \\
& +\left[c_{13}\left(\phi_{a}^{\dagger} \phi_{s}\right) \chi_{a}^{*} \chi_{s}+c_{14}\left(\phi_{s}^{\dagger} \phi_{a}\right) \xi^{*} \chi_{s}+c_{15}\left(\phi_{a}^{\dagger} \phi_{s}\right) \xi^{*} \chi_{a}+c_{16}\left(\phi_{a}^{\dagger} \phi_{a}^{\prime}\right) \chi_{a}^{*} \chi_{s}^{*}+c_{17}\left(\phi_{s}^{\prime \dagger} \phi_{a}\right) \chi_{s} \xi\right. \\
& +c_{18}\left(\phi_{s}^{\dagger} \phi_{a}^{\prime}\right) \chi_{a}^{*} \xi^{*}+c_{19}\left(\phi_{a}^{\prime \dagger} \phi_{s}\right) \chi_{s} \chi_{s}+c_{20}\left(\phi_{s}^{\prime \dagger} \phi_{s}\right) \chi_{a} \chi_{s}+c_{21}\left(\phi_{a}^{\prime \dagger} \phi_{s}^{\prime}\right) \xi^{*} \chi_{a}+c_{22}\left(\phi_{a}^{\prime \dagger} \phi_{s}^{\prime}\right) \chi_{a}^{*} \chi_{s} \\
& +c_{23}\left(\phi_{a}^{\prime \dagger} \phi_{s}^{\prime}\right) \chi_{s}^{*} \xi+c_{24}\left(\phi_{s}^{\prime \dagger} \phi_{a}\right) \chi_{a} \chi_{a}+c_{25}\left(\phi_{a}^{\dagger} \phi_{a}^{\prime}\right) \xi^{*} \xi^{*}+c_{26}\left(\phi_{s}^{\dagger} \phi_{s}^{\prime}\right) \xi^{*} \xi^{*}+\text { H.c.] } \\
& +d_{1} \xi^{*} \xi \chi_{a}^{*} \chi_{a}+d_{2} \xi^{*} \xi \chi_{s}^{*} \chi_{s}+d_{3} \chi_{s}^{*} \chi_{s} \chi_{a}^{*} \chi_{a}+\left[d_{4} \xi^{*} \xi^{*} \chi_{a} \chi_{s}+d_{5} \xi^{*} \chi_{a}^{*} \chi_{s} \chi_{s}+d_{6} \xi^{*} \chi_{s}^{*} \chi_{a} \chi_{a}+\text { H.c. }\right]
\end{aligned}
$$

In the above equation, the parameters in the first eight terms have mass-squared dimensions and the $a$ parameters have dimensions of mass. Rest of the parameters in Eq. (B1) are dimensionless. After minimizing the above scalar potential, different scalar fields of the model acquire vevs, which satisfy the following relations.

$$
\begin{aligned}
& m_{H}^{2}+2 \lambda_{H}\left\langle H^{\dagger} H\right\rangle+\lambda_{\phi_{a} H}\left\langle\phi_{a}^{\dagger} \phi_{a}\right\rangle+\lambda_{\phi_{s} H}\left\langle\phi_{s}^{\dagger} \phi_{s}\right\rangle+\lambda_{\phi_{a}^{\prime} H}\left\langle\phi_{a}^{\prime \dagger} \phi_{a}^{\prime}\right\rangle+\lambda_{\phi_{s}^{\prime} H}\left\langle\phi_{s}^{\prime \dagger} \phi_{s}^{\prime}\right\rangle \\
& +\lambda_{\chi_{a} H}\left\langle\chi_{a}^{*} \chi_{a}\right\rangle+\lambda_{\chi_{s} H}\left\langle\chi_{s}^{*} \chi_{s}\right\rangle+\lambda_{\xi H}\left\langle\xi^{*} \xi\right\rangle=0, \\
& {\left[m_{\chi_{a}}^{2}+2 \lambda_{\chi_{a}}\left\langle\chi_{a}^{*} \chi_{a}\right\rangle+c_{5}\left\langle\phi_{a}^{\dagger} \phi_{a}\right\rangle+c_{6}\left\langle\phi_{s}^{\dagger} \phi_{s}\right\rangle+c_{7}\left\langle\phi_{a}^{\prime \dagger} \phi_{a}^{\prime}\right\rangle+c_{8}\left\langle\phi_{s}^{\prime \dagger} \phi_{s}^{\prime}\right\rangle+d_{3}\left\langle\chi_{s}^{*} \chi_{s}\right\rangle+d_{1}\left\langle\xi^{*} \xi\right\rangle\right.} \\
& \left.+\lambda_{\chi_{a} H}\left\langle H^{\dagger} H\right\rangle\right]\left\langle\chi_{a}^{*}\right\rangle+a_{2}\left\langle\phi_{a}^{\dagger} \phi_{s}^{\prime}\right\rangle+\left[c_{13}^{*}\left\langle\phi_{s}^{\dagger} \phi_{a}\right\rangle+c_{22}^{*}\left\langle\phi_{s}^{\prime \dagger} \phi_{a}^{\prime}\right\rangle+d_{5}^{*}\left\langle\xi \chi_{s}^{*}\right\rangle\right]\left\langle\chi_{s}^{*}\right\rangle+\left[c_{15}\left\langle\phi_{a}^{\dagger} \phi_{s}\right\rangle\right. \\
& \left.+c_{21}\left\langle\phi_{a}^{\prime \dagger} \phi_{s}^{\prime}\right\rangle\right]\left\langle\xi^{*}\right\rangle+c_{18}^{*}\left\langle\phi_{a}^{\prime \dagger} \phi_{s}\right\rangle\langle\xi\rangle+\left[a_{5}\langle\xi\rangle+c_{16}^{*}\left\langle\phi_{a}^{\prime \dagger} \phi_{a}\right\rangle+c_{20}\left\langle\phi_{s}^{\prime \dagger} \phi_{s}\right\rangle+d_{4}\left\langle\xi^{*} \xi^{*}\right\rangle\right]\left\langle\chi_{s}\right\rangle \\
& +\left[2 c_{24}\left\langle\phi_{s}^{\prime \dagger} \phi_{a}\right\rangle+2 d_{6}\left\langle\xi^{*} \chi_{s}^{*}\right\rangle\right]\left\langle\chi_{a}\right\rangle=0, \\
& {\left[m_{\chi_{s}}^{2}+2 \lambda_{\chi_{s}}\left\langle\chi_{s}^{*} \chi_{s}\right\rangle+c_{9}\left\langle\phi_{a}^{\dagger} \phi_{a}\right\rangle+c_{10}\left\langle\phi_{s}^{\dagger} \phi_{s}\right\rangle+c_{11}\left\langle\phi_{a}^{\prime \dagger} \phi_{a}^{\prime}\right\rangle+c_{12}\left\langle\phi_{s}^{\prime \dagger} \phi_{s}^{\prime}\right\rangle+d_{2}\left\langle\xi^{*} \xi\right\rangle\right.} \\
& \left.+d_{3}\left\langle\chi_{a}^{*} \chi_{a}\right\rangle+\lambda_{x_{s} H}\left\langle H^{\dagger} H\right\rangle\right]\left\langle\chi_{s}^{*}\right\rangle+a_{3}\left\langle\phi_{s}^{\dagger} \phi_{a}^{\prime}\right\rangle+\left[c_{13}\left\langle\phi_{a}^{\dagger} \phi_{s}\right\rangle+c_{22}\left\langle\phi_{a}^{\prime \dagger} \phi_{s}^{\prime}\right\rangle\right]\left\langle\chi_{a}^{*}\right\rangle \\
& +\left[c_{14}\left\langle\phi_{s}^{\dagger} \phi_{a}\right\rangle+c_{23}^{*}\left\langle\phi_{s}^{\prime \dagger} \phi_{a}^{\prime}\right\rangle\right]\left\langle\xi^{*}\right\rangle+\left[a_{5}\langle\xi\rangle+c_{16}^{*}\left\langle\phi_{a}^{\prime \dagger} \phi_{a}\right\rangle+c_{20}\left\langle\phi_{s}^{\prime \dagger} \phi_{s}\right\rangle+d_{4}\left\langle\xi^{*} \xi^{*}\right\rangle\right]\left\langle\chi_{a}\right\rangle \\
& +\left[c_{17}\left\langle\phi_{s}^{\prime \dagger} \phi_{a}\right\rangle+d_{6}^{*}\left\langle\chi_{a}^{*} \chi_{a}^{*}\right\rangle\right]\langle\xi\rangle+\left[3 a_{8}\left\langle\chi_{s}\right\rangle+2 c_{19}\left\langle\phi_{a}^{\prime \dagger} \phi_{s}\right\rangle+2 d_{5}\left\langle\xi^{*} \chi_{a}^{*}\right\rangle\right]\left\langle\chi_{s}\right\rangle=0, \\
& {\left[m_{\xi}^{2}+2 \lambda_{\xi}\left\langle\xi^{*} \xi\right\rangle+c_{1}\left\langle\phi_{a}^{\dagger} \phi_{a}\right\rangle+c_{2}\left\langle\phi_{s}^{\dagger} \phi_{s}\right\rangle+c_{3}\left\langle\phi_{a}^{\prime \dagger} \phi_{a}^{\prime}\right\rangle+c_{4}\left\langle\phi_{s}^{\prime \dagger} \phi_{s}^{\prime}\right\rangle+d_{1}\left\langle\chi_{a}^{*} \chi_{a}\right\rangle+d_{2}\left\langle\chi_{s}^{*} \chi_{s}\right\rangle\right.} \\
& \left.+\lambda_{\xi H}\left\langle H^{\dagger} H\right\rangle\right]\left\langle\xi^{*}\right\rangle+a_{1}\left\langle\phi_{a}^{\dagger} \phi_{a}^{\prime}\right\rangle+a_{4}\left\langle\phi_{s}^{\dagger} \phi_{s}^{\prime}\right\rangle+\left[c_{14}^{*}\left\langle\phi_{a}^{\dagger} \phi_{s}\right\rangle+c_{23}\left\langle\phi_{a}^{\prime \dagger} \phi_{s}^{\prime}\right\rangle+d_{5}^{*}\left\langle\chi_{a} \chi_{s}^{*}\right\rangle\right]\left\langle\chi_{s}^{*}\right\rangle \\
& +\left[c_{15}^{*}\left\langle\phi_{s}^{\dagger} \phi_{a}\right\rangle+c_{21}^{*}\left\langle\phi_{s}^{\prime \dagger} \phi_{a}^{\prime}\right\rangle\right]\left\langle\chi_{a}^{*}\right\rangle+\left[c_{17}\left\langle\phi_{s}^{\prime \dagger} \phi_{a}\right\rangle+d_{6}^{*}\left\langle\chi_{a}^{*} \chi_{a}^{*}\right\rangle+a_{5}\left\langle\chi_{a}\right\rangle\right]\left\langle\chi_{s}\right\rangle+c_{18}^{*}\left\langle\phi_{a}^{\prime \dagger} \phi_{s}\right\rangle\left\langle\chi_{a}\right\rangle \\
& +\left[3 a_{6}\langle\xi\rangle+2 c_{25}^{*}\left\langle\phi_{a}^{\prime \dagger} \phi_{a}\right\rangle+2 c_{26}^{*}\left\langle\phi_{s}^{\prime \dagger} \phi_{s}\right\rangle+2 d_{4}^{*}\left\langle\chi_{a}^{*} \chi_{s}^{*}\right\rangle\right]\langle\xi\rangle=0,
\end{aligned}
$$




$$
\begin{aligned}
& {\left[m_{\phi_{a}}^{2}+2 \lambda_{\phi_{a}}\left\langle\phi_{a}^{\dagger} \phi_{a}\right\rangle+b_{1}\left\langle\phi_{s}^{\dagger} \phi_{s}\right\rangle+b_{2}\left\langle\phi_{a}^{\prime \dagger} \phi_{a}^{\prime}\right\rangle+b_{3}\left\langle\phi_{s}^{\prime \dagger} \phi_{s}^{\prime}\right\rangle+c_{1}\left\langle\xi^{*} \xi\right\rangle+c_{5}\left\langle\chi_{a}^{*} \chi_{a}\right\rangle+c_{9}\left\langle\chi_{s}^{*} \chi_{s}\right\rangle\right.} \\
& \left.+\lambda_{\phi_{a} H}\left\langle H^{\dagger} H\right\rangle\right]\left\langle\phi_{a}^{\dagger}\right\rangle+\left[a_{1}^{*}\left\langle\xi^{*}\right\rangle+b_{10}\left\langle\phi_{s}^{\dagger} \phi_{s}^{\prime}\right\rangle+b_{14}\left\langle\phi_{a}^{\dagger} \phi_{a}^{\prime}\right\rangle+c_{16}^{*}\left\langle\chi_{a} \chi_{s}\right\rangle+c_{25}^{*}\langle\xi \xi\rangle\right]\left\langle\phi_{a}^{\prime \dagger}\right\rangle \\
& +\left[b_{8}\left\langle\phi_{a}^{\dagger} \phi_{s}\right\rangle+b_{9}\left\langle\phi_{a}^{\prime \dagger} \phi_{s}^{\prime}\right\rangle+c_{13}^{*}\left\langle\chi_{s}^{*} \chi_{a}\right\rangle+c_{14}\left\langle\xi^{*} \chi_{s}\right\rangle+c_{15}^{*}\left\langle\chi_{a}^{*} \xi\right\rangle\right]\left\langle\phi_{s}^{\dagger}\right\rangle+\left[a_{2}^{*}\left\langle\chi_{a}^{*}\right\rangle+b_{7}\left\langle\phi_{a}^{\dagger} \phi_{s}^{\prime}\right\rangle\right. \\
& \left.+c_{17}\left\langle\chi_{s} \xi\right\rangle+c_{24}\left\langle\chi_{a} \chi_{a}\right\rangle\right]\left\langle\phi_{s}^{\prime \dagger}\right\rangle=0 \text {, } \\
& {\left[m_{\phi_{s}}^{2}+2 \lambda_{\phi_{s}}\left\langle\phi_{s}^{\dagger} \phi_{s}\right\rangle+b_{1}\left\langle\phi_{a}^{\dagger} \phi_{a}\right\rangle+b_{4}\left\langle\phi_{a}^{\prime \dagger} \phi_{a}^{\prime}\right\rangle+b_{5}\left\langle\phi_{s}^{\prime \dagger} \phi_{s}^{\prime}\right\rangle+c_{2}\left\langle\xi^{*} \xi\right\rangle+c_{6}\left\langle\chi_{a}^{*} \chi_{a}\right\rangle+c_{10}\left\langle\chi_{s}^{*} \chi_{s}\right\rangle\right.} \\
& \left.+\lambda_{\phi_{s} H}\left\langle H^{\dagger} H\right\rangle\right]\left\langle\phi_{s}^{\dagger}\right\rangle+\left[a_{3}^{*}\left\langle\chi_{s}^{*}\right\rangle+b_{11}\left\langle\phi_{s}^{\dagger} \phi_{a}^{\prime}\right\rangle+c_{18}^{*}\left\langle\chi_{a} \xi\right\rangle+c_{19}\left\langle\chi_{s} \chi_{s}\right\rangle\right]\left\langle\phi_{a}^{\prime \dagger}\right\rangle+\left[a_{4}^{*}\left\langle\xi^{*}\right\rangle\right. \\
& \left.+b_{10}^{*}\left\langle\phi_{a}^{\dagger} \phi_{a}^{\prime}\right\rangle+b_{12}\left\langle\phi_{s}^{\dagger} \phi_{s}^{\prime}\right\rangle+c_{20}\left\langle\chi_{a} \chi_{s}\right\rangle+c_{26}^{*}\langle\xi \xi\rangle\right]\left\langle\phi_{s}^{\prime \dagger}\right\rangle+\left[b_{8}\left\langle\phi_{s}^{\dagger} \phi_{a}\right\rangle+b_{9}^{*}\left\langle\phi_{s}^{\prime \dagger} \phi_{a}^{\prime}\right\rangle+c_{13}\left\langle\chi_{a}^{*} \chi_{s}\right\rangle\right. \\
& +c_{14}^{*}\left\langle\chi_{s}^{*} \xi\right\rangle+c_{15}\left\langle\xi^{*} \chi_{a}\right]\left\langle\phi_{a}^{\dagger}\right\rangle=0 \text {, } \\
& {\left[m_{\phi_{a}^{\prime}}^{2}+2 \lambda_{\phi_{a}^{\prime}}\left\langle\phi_{a}^{\prime \dagger} \phi_{a}^{\prime}\right\rangle+b_{2}\left\langle\phi_{a}^{\dagger} \phi_{a}\right\rangle+b_{4}\left\langle\phi_{s}^{\dagger} \phi_{s}\right\rangle+b_{6}\left\langle\phi_{s}^{\prime \dagger} \phi_{s}^{\prime}\right\rangle+c_{3}\left\langle\xi^{*} \xi\right\rangle+c_{7}\left\langle\chi_{a}^{*} \chi_{a}\right\rangle+c_{11}\left\langle\chi_{s}^{*} \chi_{s}\right\rangle\right.} \\
& \left.+\lambda_{\phi_{a}^{\prime} H}\left\langle H^{\dagger} H\right\rangle\right]\left\langle\phi_{a}^{\prime \dagger}\right\rangle+\left[a_{1}\langle\xi\rangle+b_{10}^{*}\left\langle\phi_{s}^{\prime \dagger} \phi_{s}\right\rangle+b_{14}\left\langle\phi_{a}^{\prime \dagger} \phi_{a}\right\rangle+c_{16}\left\langle\chi_{a}^{*} \chi_{s}^{*}\right\rangle+c_{25}\left\langle\xi^{*} \xi^{*}\right\rangle\right]\left\langle\phi_{a}^{\dagger}\right\rangle \\
& +\left[a_{3}\left\langle\chi_{s}\right\rangle+b_{11}\left\langle\phi_{a}^{\prime \dagger} \phi_{s}\right\rangle+c_{18}\left\langle\chi_{a}^{*} \xi^{*}\right\rangle+c_{19}^{*}\left\langle\chi_{s}^{*} \chi_{s}^{*}\right\rangle\right]\left\langle\phi_{s}^{\dagger}\right\rangle+\left[b_{13}\left\langle\phi_{a}^{\prime \dagger} \phi_{s}^{\prime}\right\rangle+b_{9}^{*}\left\langle\phi_{a}^{\dagger} \phi_{s}\right\rangle+c_{21}^{*}\left\langle\xi \chi_{a}^{*}\right\rangle\right. \\
& \left.+c_{22}^{*}\left\langle\chi_{a} \chi_{s}^{*}\right\rangle+c_{23}^{*}\left\langle\chi_{s} \xi^{*}\right\rangle\right]\left\langle\phi_{s}^{\prime \dagger}\right\rangle=0 \text {, } \\
& {\left[m_{\phi_{s}^{\prime}}^{2}+2 \lambda_{\phi_{s}^{\prime}}\left\langle\phi_{s}^{\prime \dagger} \phi_{s}^{\prime}\right\rangle+b_{3}\left\langle\phi_{a}^{\dagger} \phi_{a}\right\rangle+b_{5}\left\langle\phi_{s}^{\dagger} \phi_{s}\right\rangle+b_{6}\left\langle\phi_{a}^{\prime \dagger} \phi_{a}^{\prime}\right\rangle+c_{4}\left\langle\xi^{*} \xi\right\rangle+c_{8}\left\langle\chi_{a}^{*} \chi_{a}\right\rangle+c_{12}\left\langle\chi_{s}^{*} \chi_{s}\right\rangle\right.} \\
& \left.+\lambda_{\phi_{s}^{\prime} H}\left\langle H^{\dagger} H\right\rangle\right]\left\langle\phi_{s}^{\prime \dagger}\right\rangle+\left[a_{2}\left\langle\chi_{a}\right\rangle+b_{7}\left\langle\phi_{s}^{\prime \dagger} \phi_{a}\right\rangle+c_{17}^{*}\left\langle\chi_{s}^{*} \xi^{*}\right\rangle+c_{24}^{*}\left\langle\chi_{a}^{*} \chi_{a}^{*}\right\rangle\right]\left\langle\phi_{a}^{\dagger}\right\rangle+\left[a_{4}\langle\xi\rangle+b_{10}\left\langle\phi_{a}^{\prime \dagger} \phi_{a}\right\rangle\right. \\
& \left.+b_{12}\left\langle\phi_{s}^{\prime \dagger} \phi_{s}\right\rangle+c_{20}^{*}\left\langle\chi_{a}^{*} \chi_{s}^{*}\right\rangle+c_{26}\left\langle\xi^{*} \xi^{*}\right\rangle\right]\left\langle\phi_{s}^{\dagger}\right\rangle+\left[b_{9}\left\langle\phi_{s}^{\dagger} \phi_{a}\right\rangle+b_{13}\left\langle\phi_{s}^{\prime \dagger} \phi_{a}^{\prime}\right\rangle+c_{21}\left\langle\xi^{*} \chi_{a}\right\rangle+c_{22}\left\langle\chi_{a}^{*} \chi_{s}\right\rangle\right. \\
& \left.+c_{23}\left\langle\chi_{s}^{*} \xi\right\rangle\right]\left\langle\phi_{a}^{\prime \dagger}\right\rangle=0 \text {. }
\end{aligned}
$$

In Sec. VI, in order to obtain correct phenomenology in the neutrino sector, the order magnitude of the vevs of different scalar fields of our model have been estimated. Since these vevs are determined by the Eqs. (B2) - (B9), one has to adjust the unknown parameters of these relations in such a way that the above mentioned order of magnitude for these vevs can be obtained. In order to fix these parameters, we first assume that the mass-square parameters in Eq. (B1) should be around the square of the vevs of the corresponding fields. This assumption is based on the fact that after spontaneous symmetry breaking, a scalar field acquires mass around the scale at which the symmetry is broken. As a result of this, we take the scales of the masssquare parameters as:

$$
\begin{aligned}
& m_{H}^{2} \sim(100 \mathrm{GeV})^{2}, \quad m_{\chi_{a}}^{2}, \quad m_{\chi_{s}}^{2} \sim(1 \mathrm{TeV})^{2}, \\
& m_{\phi_{a}}^{2}, \quad m_{\phi_{s}}^{2}, \quad m_{\phi_{a}^{\prime}}^{2}, \quad m_{\phi_{s}^{\prime}}^{2} \sim\left(10^{12} \mathrm{GeV}\right)^{2}, \\
& m_{\xi}^{2} \sim\left(10^{17} \mathrm{GeV}\right)^{2} .
\end{aligned}
$$

Now, in order to achieve the desired magnitude of the vevs for the scalar fields, we can estimate the unknown parameters of Eqs. (B2) - (B9). These are given below.
$\lambda_{H}, \lambda_{\chi_{a}}, \lambda_{\chi_{s}}, \lambda_{\xi}, \lambda_{\phi_{a}}, \lambda_{\phi_{s}}, \lambda_{\phi_{a}^{\prime}}, \lambda_{\phi_{s}^{\prime}} \sim 1$,

$\lambda_{\phi_{a} H}, \lambda_{\phi_{s} H}, \lambda_{\phi_{a}^{\prime} H}, \lambda_{\phi_{s}^{\prime} H} \sim 10^{-20}$,

$\lambda_{\chi_{a} H}, \lambda_{\chi_{s} H} \sim 10^{-2}, \quad \lambda_{\xi H} \sim 10^{-30}$,

$a_{1}, a_{4} \sim 10^{7} \mathrm{GeV}, \quad a_{2}, a_{3} \sim 10^{-15} \mathrm{GeV}$,

$a_{5} \sim 10^{-11} \mathrm{GeV}, \quad a_{6} \sim 10^{17} \mathrm{GeV}$,

$a_{7}, a_{8} \sim 10^{3} \mathrm{GeV}, \quad b_{1}, \ldots, b_{14} \sim 1$,

$c_{1}, c_{2}, c_{3}, c_{4}, c_{25}, c_{26} \sim 10^{-10}$,

$c_{5}, c_{6}, c_{7}, c_{8}, c_{9}, c_{10}, c_{11}, c_{12}, c_{13}, c_{16}, c_{19}, c_{20}, c_{22}, c_{24} \sim 10^{-18}$,

$c_{14}, c_{15}, c_{17}, c_{18}, c_{21}, c_{23} \sim 10^{-32}$,

$d_{1}, d_{2}, d_{4} \sim 10^{-28}, \quad d_{3} \sim 1, \quad d_{5}, d_{6} \sim 10^{-14}$.

From the above mentioned values for the unknown parameters, we notice that some couplings need to be suppressed in order to achieve the desired hierarchy in the vevs of the scalar fields of our model.

Some parameters in the potential of Eq. (B1) can be complex. As a result of this, after solving Eqs. (B2) - (B9), except for the Higgs field, rest of the fields can acquire complex vevs. We will explain about the vacuum alignment for $\phi_{a}$ and $\phi_{s}$ shortly below. Assuming this vacuum alignment, using the above mentioned complex vevs in 
our model of previous section, Dirac and Majorana mass matrices for neutrinos are generated with complex elements. Hence, the following parameters are complex: $a, e$, $\epsilon_{i}, M_{\mathrm{atm}}$, and $M_{\mathrm{sol}}$. With these complex parameters, we can explain the neutrino masses and mixing angles and it is described in Sec. IV that the phases of these parameters can be fixed in order to get real values for the neutrino masses. On the other hand, to explain the neutrino oscillation data, it is sufficient to make $\epsilon_{i}$ to be complex and rest of the above mentioned parameters can be chosen to be real. To achieve this particular case, we choose the following parameters of Eq. (B1) to be complex: $a_{1}, a_{4}, a_{5}, a_{6}, c_{14}, c_{15}, c_{17}, c_{18}, c_{21}, c_{23}, c_{25}, c_{26}, d_{4}, d_{5}, d_{6}$. The phases of these parameters can be adjusted in such a way that only $\langle\xi\rangle$ can be complex and rest of the scalar fields have real vevs. As a result of this, only $\epsilon_{i}$ become complex and rest of the parameters of Dirac and Majorana mass matrices can be real. It is explained before that the scalar fields $\phi_{a}$ and $\phi_{s}$ need to acquire vevs in some particular directions, in order to obtain TBM pattern in the neutrino sector. The vev for these fields are determined by solving Eqs. (B6) - (B9). The unknown parameters in these equations should be fine-tuned in such a way that the vevs for $\phi_{a}$ and $\phi_{s}$ can acquire the desired directions. One can notice that, in order to do this fine-tuning, enough number of parameters exist in Eqs. (B6) - (B9). Hence, in our scenario, the desired vacuum alignment for $\phi_{a}$ and $\phi_{s}$ can be achieved. The scalar potential given in Eq. (B1) is at tree level. This potential can get corrections at loop level. Since loop effects give small corrections to the tree level potential and due to large number of parameters in our model, we can still fine-tune these parameters in order to get the necessary vacuum alignment for the above scalar fields.
[1] M. C. Gonzalez-Garcia and M. Maltoni, Phys. Rep. 460, 1 (2008).

[2] P. Minkowski, Phys. Lett. 67B, 421 (1977); T. Yanagida, in Proceedings of the Workshop on the Unified Theory and the Baryon Number in the Universe, edited by O. Sawada and A. Sugamoto (KEK, Tsukuba, Japan, 1979), p. 95; M. Gell-Mann, P. Ramond, and R. Slansky, Complex spinors and unified theories, in Supergravity, edited by P. van Nieuwenhuizen and D. Z. Freedman (North Holland, Amsterdam, 1979), p. 315; S. L. Glashow, The future of elementary particle physics, in Proceedings of the 1979 Cargèse Summer Institute on Quarks and Leptons, edited by M. Lévy, J.-L. Basdevant, D. Speiser, J. Weyers, R. Gastmans, and M. Jacob (Plenum Press, New York, 1980), pp. 687-713.

[3] R. N. Mohapatra and G. Senjanovic, Phys. Rev. Lett. 44, 912 (1980); J. Schechter and J. W. F. Valle, Phys. Rev. D 22, 2227 (1980).

[4] S. F. King, Phys. Lett. B 439, 350 (1998); Nucl. Phys. B562, 57 (1999); B576, 85 (2000); S. F. KingJ. High Energy Phys. 09 (2002) 011; T. Blazek and S. F. King, Nucl. Phys. B662, 359 (2003).

[5] S. Antusch, S. Boudjemaa, and S. F. King, J. High Energy Phys. 09 (2010) 096.

[6] S. F. King, J. High Energy Phys. 08 (2005) 105.

[7] S. Antusch, S. F. King, C. Luhn, and M. Spinrath, Nucl. Phys. B856, 328 (2012); S. F. King, J. High Energy Phys. 07 (2013) 137; 02 (2016) 085; S. F. King and C. Luhn, J. High Energy Phys. 09 (2016) 023; P. Ballett, S. F. King, S. Pascoli, N. W. Prouse, and T. Wang, J. High Energy Phys. 03 (2017) 110; S. F. King, S. Molina Sedgwick, and S. J. Rowley, J. High Energy Phys. 10 (2018) 184; S. F. King, Phys. Lett. B 724, 92 (2013); J. High Energy Phys. 01 (2014) 119; F. Bjrkeroth and S. F. King, J. Phys. G 42, 125002 (2015).
[8] I. Esteban, M. C. Gonzalez-Garcia, A. HernandezCabezudo, M. Maltoni, and T. Schwetz, J. High Energy Phys. 01 (2019) 106.

[9] P. F. Harrison, D. H. Perkins, and W. G. Scott, Phys. Lett. B 530, 167 (2002); P. F. Harrison and W. G. Scott, Phys. Lett. B 535, 163 (2002); Z.-Z. Xing, Phys. Lett. B 533, 85 (2002).

[10] F. P. An et al. (Daya Bay Collaboration), Phys. Rev. Lett. 108, 171803 (2012); J. K. Ahn et al. (RENO Collaboration), Phys. Rev. Lett. 108, 191802 (2012).

[11] R. S. Hundi and I. Sethi, Phys. Rev. D 102, 055007 (2020).

[12] S. F. King, J. High Energy Phys. 09 (2002) 011; Phys. Rev. D 67, 113010 (2003).

[13] M. Tanabashi et al. (Particle Data Group), Phys. Rev. D 98, 030001 (2018).

[14] S. F. King, Phys. Lett. B 659, 244 (2008); S. F. King, C. Luhn, and A. J. Stuart, Nucl. Phys. B867, 203 (2013).

[15] S. Pakvasa, W. Rodejohann, and T. J. Weiler, Phys. Rev. Lett. 100, 111801 (2008).

[16] S. F. King, Phys. Lett. B 675, 347 (2009).

[17] P. F. de Salas, D. V. Forero, S. Gariazzo, P. Martínez-Miravé, O. Mena, C. A. Ternes, M. Tórtola, and J. W. F. Valle, arXiv: 2006.11237.

[18] H. Baer and X. Tata, Weak Scale Supersymmetry: From Superfields to Scattering Events (Cambridge University Press, Cambridge, England, 2006); M. Drees, R. Godbole, and P. Roy, Theory and Phenomenology of S Particles (World Scientific, Singapore, 2004); P. Bińetruy, Supersymmetry (Oxford University Press, Oxford, England, 2006); S. P. Martin, Adv. Ser. Dir. High Energy Phys. 21, 1 (2010).

[19] N. Arkani-Hamed, L. J. Hall, H. Murayama, D. TuckerSmith, and N. Weiner, Phys. Rev. D 64, 115011 (2001); F. Borzumati and Y. Nomura, Phys. Rev. D 64, 053005 (2001). 